\title{
Germinal center dysregulation by histone methyltransferase EZH2 promotes lymphomagenesis
}

\author{
Marieta Caganova, ${ }^{1}$ Chiara Carrisi, ${ }^{1}$ Gabriele Varano, ${ }^{1}$ Federica Mainoldi, ${ }^{1}$ Federica Zanardi, ${ }^{1}$ \\ Pierre-Luc Germain, ${ }^{2}$ Laura George, ${ }^{3}$ Federica Alberghini, ${ }^{1}$ Luca Ferrarini, ${ }^{1}$ \\ Asoke K. Talukder, ${ }^{4}$ Maurilio Ponzoni, ${ }^{5}$ Giuseppe Testa, ${ }^{2}$ Takuya Nojima, ${ }^{6}$ Claudio Doglioni, ${ }^{5}$ \\ Daisuke Kitamura, ${ }^{6}$ Kai-M. Toellner, ${ }^{3}$ I-hsin Su, ${ }^{7}$ and Stefano Casola ${ }^{1}$
}

\begin{abstract}
${ }^{1}$ The Institute of Molecular Oncology (IFOM) of the Italian Foundation for Cancer Research (FIRC), Milan, Italy.
2European Institute of Oncology, Milan, Italy. ${ }^{3}$ Medical Research Council Centre for Immune Regulation, School of Immunity and Infection, University of Birmingham, Birmingham, United Kingdom. ${ }^{4}$ InterpretOmics India, Bangalore, India. ${ }^{5}$ Unit of Lymphoid Malignancies, Department of Onco-Haematology, San Raffaele Scientific Institute, Milan, Italy. ${ }^{6}$ Division of Molecular Biology, Research Institute for Biological Sciences, Tokyo University of Science, Noda, Chiba, Japan. ${ }^{7 D i v i s i o n ~ o f ~ M o l e c u l a r ~ G e n e t i c s ~ a n d ~ C e l l ~ B i o l o g y, ~ S c h o o l ~ o f ~ B i o l o g i c a l ~ S c i e n c e s, ~}$ Nanyang Technological University, Singapore.
\end{abstract}

\begin{abstract}
Protection against deadly pathogens requires the production of high-affinity antibodies by $B$ cells, which are generated in germinal centers (GCs). Alteration of the GC developmental program is common in many $\mathrm{B}$ cell malignancies. Identification of regulators of the GC response is crucial to develop targeted therapies for GC B cell dysfunctions, including lymphomas. The histone $\mathrm{H3}$ lysine 27 methyltransferase enhancer of zeste homolog 2 (EZH2) is highly expressed in GC B cells and is often constitutively activated in GC-derived non-Hodgkin lymphomas (NHLs). The function of EZH2 in GC B cells remains largely unknown. Herein, we show that $E z h 2$ inactivation in mouse GC B cells caused profound impairment of GC responses, memory $B$ cell formation, and humoral immunity. EZH2 protected GC B cells against activation-induced cytidine deaminase (AID) mutagenesis, facilitated cell cycle progression, and silenced plasma cell determinant and tumor suppressor B-lymphocyte-induced maturation protein 1 (BLIMP1). EZH2 inhibition in NHL cells induced BLIMP1, which impaired tumor growth. In conclusion, EZH2 sustains AID function and prevents terminal differentiation of GC B cells, which allows antibody diversification and affinity maturation. Dysregulation of the GC reaction by constitutively active EZH2 facilitates lymphomagenesis and identifies EZH2 as a possible therapeutic target in NHL and other GC-derived B cell diseases.
\end{abstract}

\begin{abstract}
Introduction
Protective immunity against pathogens relies on the production of high-affinity antibodies by long-lived plasma cells (PCs). Moreover, the ability to respond faster and with more potent antibodies to subsequent encounters with the same infectious agent depends on the generation of long-lived memory B cells. Both, high-affinity memory B cells and PCs differentiate from antigenspecific $B$ cells that are recruited into the $\mathrm{GC}$ reaction during $\mathrm{T}$ cell-dependent immune responses (1). In GCs, B cells undergo clonal expansion, a process during which they accumulate mutations at high frequency within the Ig heavy and light chain variable $(V)$ region genes. The highly dynamic nature of the GC reaction is characterized by repeated cycles of cell division, Ig somatic mutation, and strict selection based on the ability of B cells to capture and present antigen to $\mathrm{T}$ follicular helper cells (2). These processes occur within distinct areas of the GC reached by B cells through migratory paths regulated by chemokine gradients (1). The molecular determinants enabling cyclic reentry of B cells into the proliferating and mutating compartment of centroblasts, preventing terminal differentiation and the ensuing exit from the $\mathrm{GC}$, remain poorly characterized.
\end{abstract}

Authorship note: Chiara Carrisi and Gabriele Varano contributed equally to this work. Conflict of interest: The authors have declared that no conflict of interest exists. Citation for this article: J Clin Invest. 2013;123(12):5009-5022. doi:10.1172/JCI70626.
Polycomb group (PcG) proteins act within 2 main polycomb repressive complexes (PRC1 and PRC2) to promote gene silencing. PRC1 and PRC2 catalyze posttranslational modifications of specific lysine residues in core histone tails, resulting in chromatin compaction (3). Changes in chromatin conformation regulated by PcG activity represent important molecular switches that control cell differentiation, proliferation, and survival in prenatal and postnatal life (4). Enhancer of zeste homolog 2 (EZH2) is the main catalytic subunit of PRC2. Through its SET domain, EZH2 catalyzes histone $\mathrm{H} 3$ lysine 27 trimethylation (H3K27me3), which is enriched at transcription start sites (TSSs) of repressed genes (5). Together with $\mathrm{H} 3 \mathrm{~K} 4 \mathrm{me} 3, \mathrm{H} 3 \mathrm{~K} 27 \mathrm{me} 3$ is found at promoters of regulators of lineage specification, where it acts to fine-tune their expression (6).

EZH2 is expressed at high levels in human GC B cells $(7,8)$. Moreover, whole-exome sequencing efforts have revealed that $E Z H 2$ gain-of-function mutations are among the most common genetic alterations identified in diffuse large B cell lymphoma (DLBCL) and follicular lymphoma originating from GC B cells $(9,10)$. Together, these results point to a critical role of EZH2 in GC B cell function and in the pathogenesis of GC-derived nonHodgkin lymphoma (NHL).

Using GC B cell-specific gene targeting in mice, we show that EZH2 methyltransferase activity is required to protect GC B cells against genotoxic damage induced by activation-induced cytidine deaminase (AID). Moreover, we found that EZH2 is necessary to 
A

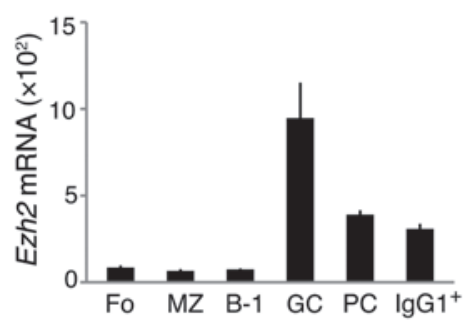

B

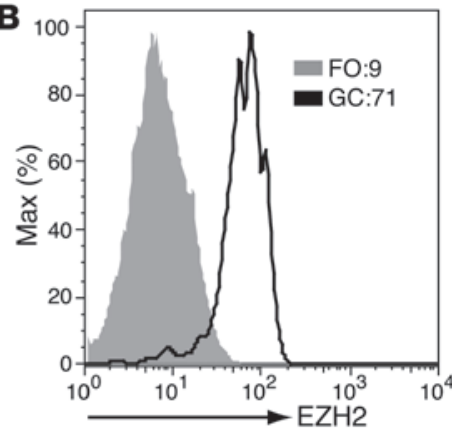

C

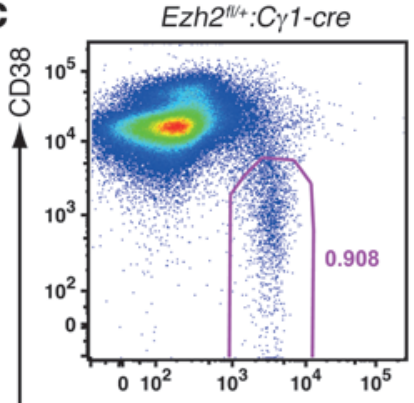

Ezh2 ${ }^{(n t)}: C \gamma 1-\mathrm{cre}$

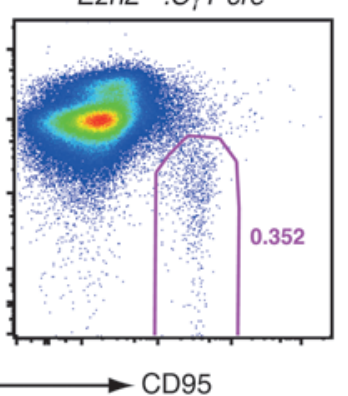

D

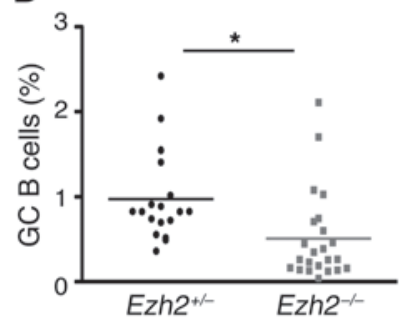

E

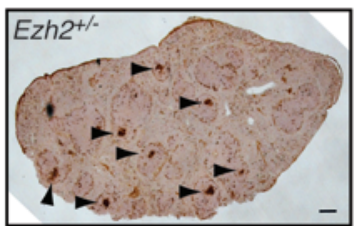

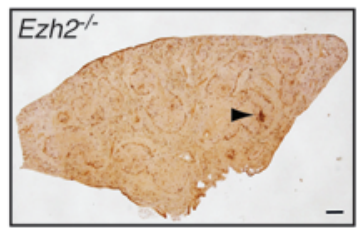

F

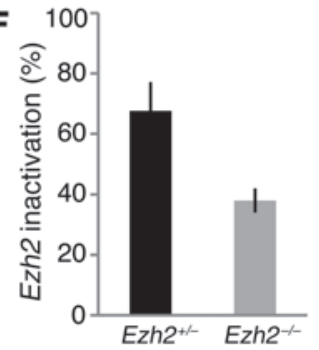

G

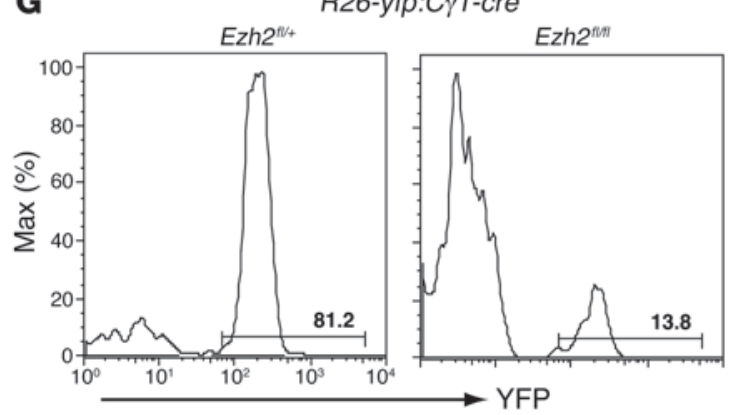

H

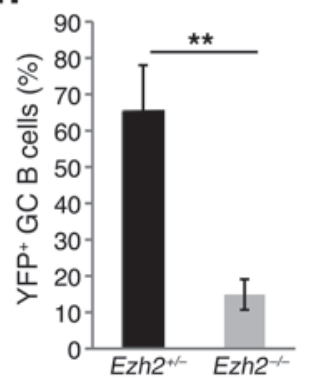

Figure 1

EZH2 is upregulated in mouse GC B cells and required for GC formation. (A) Ezh2 transcript levels in B cell subsets relative to Rplp0. Columns represent mean \pm SD of triplicates. Experiments were performed on pools of B cells sorted from 3 mice. (B) FACS analysis of EZH2 protein levels in follicular (FO) and GC B cells. Numbers indicate mean expression. (C) Representative FACS analysis of splenic B cells in NP-CGG-immunized

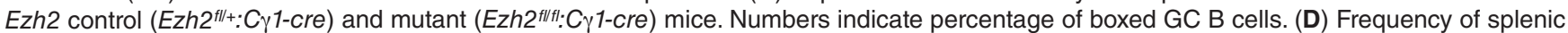

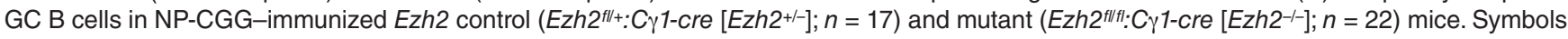
represent individual mice; bars refer to mean values. ${ }^{*} P=0.013$ ( $t$ test). (E) Representative histological analysis of PNA-positive (brown) GCs

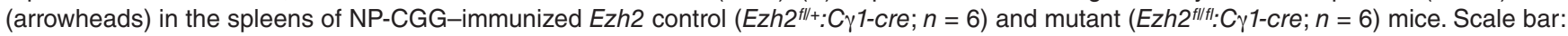
$200 \mu \mathrm{m}$. (F) Frequency of Ezh2 inactivation in GC B cells of Ezh2 control $\left(E z h 2^{f l l+}: C \gamma 1\right.$-cre; $\left.n=3\right)$ and mutant $\left(E z h 2^{f l / f l}: C \gamma 1-\right.$ cre; $\left.n=4\right)$ animals, quantified by genomic qPCR. (G) Frequency of YFP+ GC B cells in R26-yfp;Ezh2 control (Ezh2 $\left.2^{f /+}\right)$ and mutant $\left(\right.$ Ezh2 $\left.2^{f / f}\right)$ mice. (H) Summary of data on frequencies of YFP+ GC B cells in R26-yfp;C 7 1-cre; Ezh2 control $(n=4)$ and mutant $(n=6)$ mice. $(\mathbf{F}$ and $\mathbf{H})$ Columns indicate mean $\pm \mathrm{SD}$. ${ }^{\star \star} P=0.002$ ( $t$ test). Data represent $(\mathbf{C}$ and $\mathbf{D}) 10,(\mathbf{G}) 5$, (F, and $\left.\mathbf{H}\right) 3$, and $(\mathbf{B}, \mathbf{E}$, and $\mathbf{F}) 2$ experiments, respectively.

repress B-lymphocyte-induced maturation protein 1 (Blimp1) and Irf4 expression in GC B cells to limit terminal B cell differentiation induced by IL-21. Through these mechanisms, EZH2 ensures the persistence of $\mathrm{B}$ cells in the $\mathrm{GC}$ reaction, thus allowing the generation of high-affinity antibodies and memory B cells. We also found that constitutively active EZH2 is critical to stably repress tumor suppressor BLIMP1 expression in GC-type DLBCL cells, thereby possibly contributing to lymphomagenesis.

\section{Results}

Ezh2 is upregulated in mouse GC B cells. To investigate the expression of Ezh2 in mouse mature B cell subsets, we performed quantitative RT-PCR (qRT-PCR) analysis. Low Ezh2 mRNA levels were detected in follicular, marginal zone, and B-1 mature B cell subsets. A substantial increase in both Ezh2 transcript (Figure 1A) and protein (Figure 1B) levels was detected in B cells upon recruitment into the GC reaction during a $\mathrm{T}$ cell-dependent immune response. Within the GC, EZH2 was expressed predominantly in proliferating CXCR4+ centroblasts (Supplemental Figure 1A; supplemental material available online with this article; doi:10.1172/ JCI70626DS1). The differentiation of GC B cells into long-lived IgG1 ${ }^{+}$memory B cells or PCs coincided with lower, yet detectable, Ezh2 transcripts (Figure 1A). These results pointed to a role of EZH2 in GC B cell physiology.

Reduced number of GC B cells upon inducible Ezh2 inactivation. To study the specific role of EZH2 in GC B cells, conditional Ezh2 
A

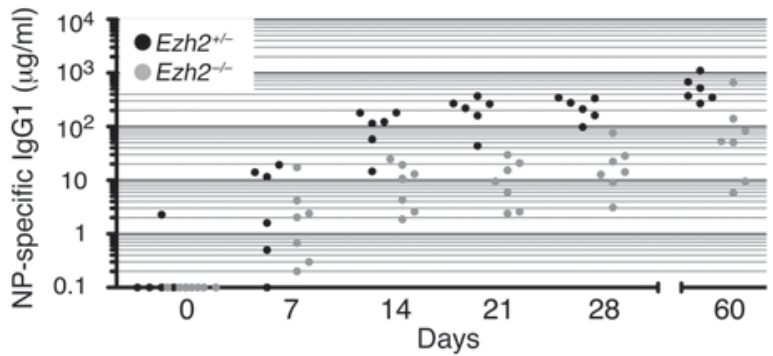

B
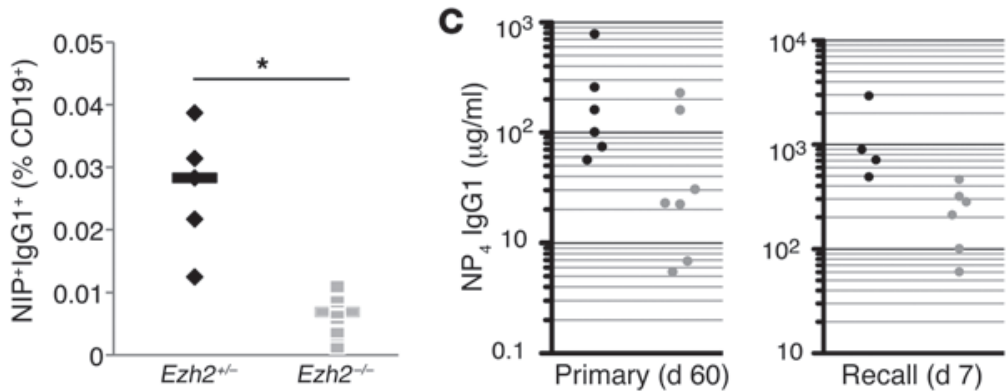

D

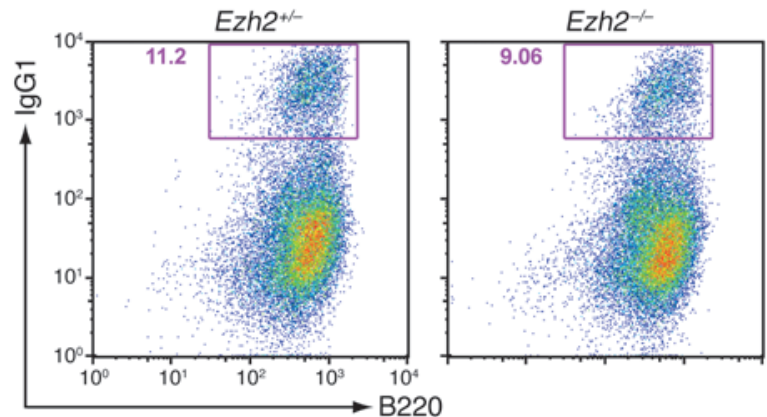

\section{Figure 2}

T cell-dependent antibody responses and memory $B$ cells depend on $\mathrm{EZH} 2$ catalytic function in $\mathrm{GC}$ B cells. (A) NP-specific IgG1 serum titers in Ezh2 control

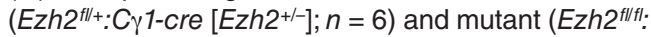
C $\gamma 1$-cre $\left.\left[E_{z h 2^{--}}\right] ; n=7\right)$ mice after primary immunization with NP-CGG. Each symbol represents 1 mouse. (B) Frequency of NP-specific lgG $1^{+}$memory

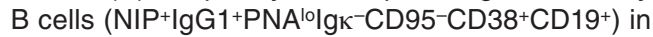
the spleens of Ezh2 control (Ezh2 ${ }^{\mathrm{fl}+}: \mathrm{C} \gamma 1$-cre; $\left.n=5\right)$ and mutant (Ezh2 ${ }^{\text {flffl: }}: C \gamma 1$-cre; $\left.n=7\right)$ mice 60 days after primary NP-CGG immunization measured by FACS. Each symbol represents 1 mouse; bars represent median values. ${ }^{*} P=0.009$ ( $t$ test). (C) NP-specific high-affinity $\left(\mathrm{NP}_{4}\right)$ IgG1 serum titers in Ezh2 control (Ezh2 ${ }^{f / l}+: C \gamma 1$-cre; $n=6$; black circles) and mutant (Ezh2 ${ }^{\mathrm{fllfl}: \mathrm{C}} \mathrm{C} \gamma 1$-cre; $n=6$; gray circles) mice at the end of the primary response (day 60) and 1 week after NPCGG boost (day 67). For the secondary response, 4 controls and 6 Ezh2 mutant mice were used, respectively. (D) FACS analysis of representative Ezh2 control (Ezh2 ${ }^{f l+}:$ Cr2-cre [Ezh2 $\left.\left.{ }^{+/}\right]\right)$and mutant

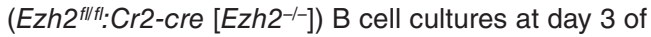
LPS+IL-4 stimulation. Numbers indicate frequency of boxed IgG1+ $B$ cells. Data are representative of $(\mathbf{A}-\mathbf{C})$ 2 and (D) 5 experiments. knockout $\left(E z h 2^{f l}\right)$ mice carrying loxP sites flanking exons coding for the catalytic SET domain (11) were crossed with $C \gamma 1$-cre knockin animals expressing Cre recombinase upon onset of Ig class switch recombination (CSR) (12). Ezh $2^{f l}$ mice were also crossed with $\mathrm{Cr}$-cre transgenic mice to inactivate $E z h 2$ in all peripheral mature B cells (13). Ezh2flffl:C $\gamma 1$-cre conditional mutants and $E z h 2^{f l+}: C \gamma 1$-cre controls (hereafter referred to as controls) were immunized with alum-precipitated 4-hydroxy-3-nitrophenyl acetyl (NP) conjugated to chicken $\gamma$ globulin (CGG) to induce $\mathrm{GC}$ responses. Flow cytometry analysis revealed an average 3 -fold reduction in percentages and absolute numbers of splenic CD $19^{+} \mathrm{CD} 38^{\mathrm{lo}} \mathrm{CD} 95^{\text {hi }} \mathrm{GC}$ B cells in Ezh2f/fl: C 1 -cre animals, 12 to 14 days after immunization, at the peak of the response (Figure 1, C and D, and Supplemental Figure 1B). Histological analysis of spleen sections of immunized mice revealed a significant reduction in peanut agglutinin (PNA)-positive GCs in Ezh2 mutant animals (Figure 1E and Supplemental Figure 1C). Similar results were observed analyzing GC responses in Ezh2flfl:Cr2-cre mutants (Supplemental Figure 1D) that showed normal distribution and number of peripheral B cell subsets (Supplemental Figure 1, E and F).

To reveal the identity of the remaining GC B cells in Ezh $2^{f l / f l}$ : C 1 -cre animals, we determined the status of the $E z h 2^{f l}$ gene by quantitative PCR (qPCR) analysis. Whereas Cre-mediated recombination occurred in the majority of $\mathrm{GC} B$ cells in

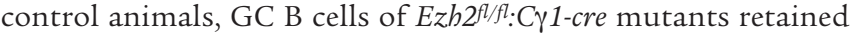
substantial levels of the unrecombined $E z h 2^{f l}$ allele (Figure 1F), giving rise to full-length Ezh2 transcripts (Supplemental Figure $1 G)$. The counter selection of Ezh2 mutant GC B cells was confirmed in Ezh2flfl:Cr2-cre mice (Supplemental Figure $1 \mathrm{H}$ ) and in animals generated from crosses of Ezh2fl:C 1 -cre mice to the R26-yfp Cre reporter strain. Flow cytometry analysis of splenic B cells isolated from immunized mice revealed a 4 - to 6 -fold reduction in the

Table 1

Mutation analysis of $V_{H} 186.2$ in Ezh2 control and mutant GC B cells

\begin{tabular}{|c|c|c|c|}
\hline Genotype & & $\begin{array}{l}\text { utation frequency } \\
(\%)\end{array}$ & \\
\hline 27 & 10 & $\%$ & 8 \\
\hline 2712 & & & \\
\hline \multicolumn{4}{|c|}{ 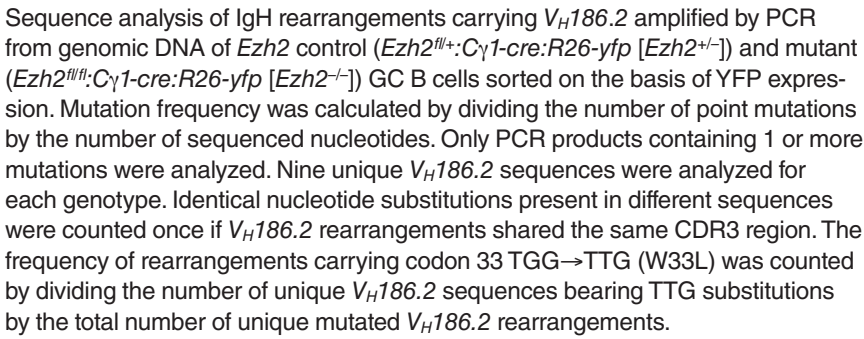 } \\
\hline
\end{tabular}


A
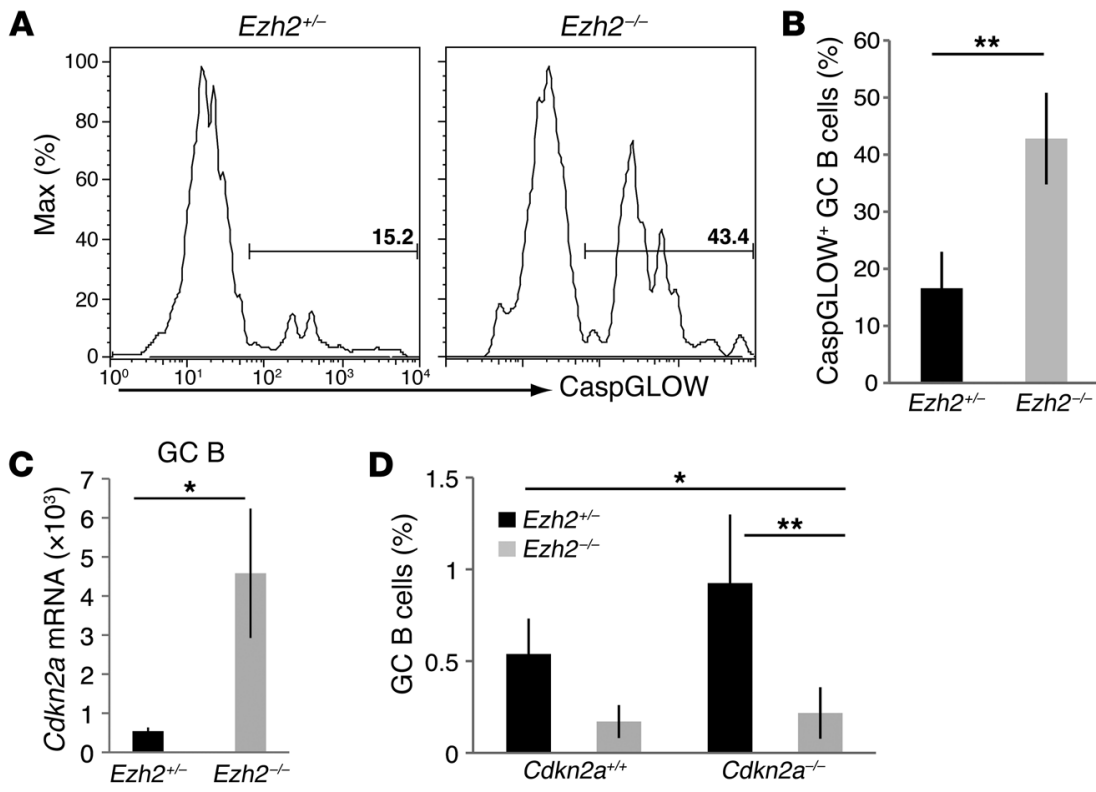

E

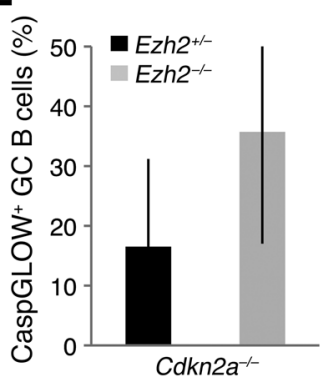

F

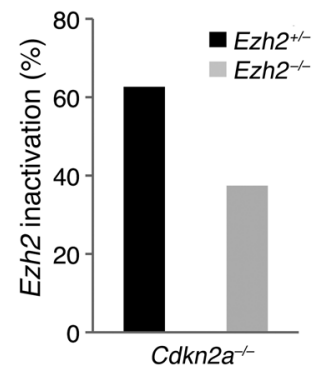

Figure 3

Ezh2 inactivation increases apoptosis of GC B cells. (A) Representative FACS analysis of CaspGLOW staining of GC B cells in the spleens of Ezh2 control (Ezh2 ${ }^{f /+}: C \gamma 1$-cre [Ezh2 $\left.\left.2^{+/}\right]\right)$and

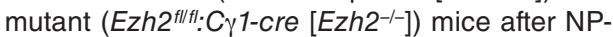
CGG immunization. Numbers indicate percentage of CaspGLOW ${ }^{+}$cells. (B) Mean frequency $\pm \mathrm{SD}$ of CaspGLOW ${ }^{+} \mathrm{GC}$ B cells in Ezh2 control $\left(E z h 2^{f l /+}: C \gamma 1\right.$-cre; $\left.n=6\right)$ and mutant (Ezh2 $2^{f / f l}$. C $\gamma 1$-cre; $n=4$ ) mice immunized with NP-CGG. ${ }^{* *} P=0.002$ ( $t$ test). (C) qRT-PCR analysis of Cdkn2a transcripts in purified GC B cells of Ezh2 control $\left(E z h 2^{f l l}:: C \gamma 1-c r e ; n=12\right)$ and mutant (Ezh2 ${ }^{f l l f l}$ : C $\gamma 1$-cre; $n=12$ ) mice after NP-CGG immunization. Values are relative to $R p / p 0$ expression. ${ }^{*} P=0.033$ ( $t$ test). (D) Frequency of splenic GC B cells in Ezh2 control (Ezh2 $\left.2^{f l+}: C \gamma 1-c r e\right)$ and mutant (Ezh2 $2^{f l / f l}$ : C $\gamma 1$-cre) mice in the presence $\left(C d k n 2 a^{+/+} ; n=5\right.$ or more) or absence (Cdkn2a ${ }^{-l-} ; n=3$ or more) of $C d k n 2 a$, as measured by flow cytometry after NP-CGG immunization. ${ }^{*} P=0.018 ;{ }^{*} P=0.0017$ ( $t$ test). (E) Proportion of CaspGLOW ${ }^{+}$splenic GC $B$ cells revealed by FACS analysis in Cdkn2adeficient Ezh2 control (Ezh2 ${ }^{\mathrm{fl}+}: \mathrm{C} \gamma 1$-cre; $\left.n=7\right)$ and mutant $\left(E z h 2^{f / l f l}: C \gamma 1\right.$-cre; $\left.n=5\right)$ mice after NP-CGG immunization. (F) Efficiency of Ezh2 inactivation in purified splenic GC B cells of NP-CGG-immunized Cdkn2a-deficient Ezh2 control (Ezh2 ${ }^{\mathrm{fl} / \mathrm{+}}: \mathrm{C} \gamma 1$-cre; $n=2)$ and mutant (Ezh2 ${ }^{f / l f l}: C \gamma 1$-cre; $\left.n=2\right)$ mice. Data are representative of (A, $\mathbf{B}$, and $\mathbf{F}) 2$, (D and E) 4, and (C) 10 experiments, respectively. (C) Columns indicate mean \pm SEM. (D and E) Columns indicate mean \pm SD. percentage of YFP ${ }^{+} \mathrm{GC} B$ cells in Ezh2 $2^{f / f l}: C \gamma 1$-cre:R26-yfp animals as compared with that in controls (Figure $1, \mathrm{G}$ and $\mathrm{H}$ ). The fraction

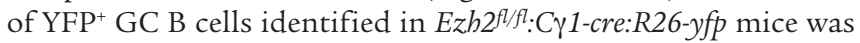
enriched for Ezh2 mutant alleles (Supplemental Figure 1I), indicating that inactivation of the PcG protein had occurred within the GC. These studies reveal that EZH2 is strictly required for the persistence of $\mathrm{B}$ cells within the GC reaction.

Poor antibody responses and fewer memory B cells upon GC B cellspecific inactivation of Ezh2. We next investigated the effects of GC $B$ cell-specific inactivation of Ezh2 on the ability of animals to mount an antigen-specific $\mathrm{T}$ cell-dependent antibody response.

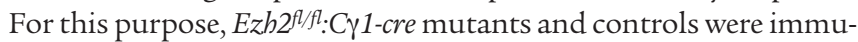
nized with NP-CGG. On average, NP-specific IgG1 titers were over 10 -fold reduced in Ezh2 mutant mice throughout the primary immune response (Figure 2A). The percentage of high-affinity NPspecific IgG $1^{+}$memory $\mathrm{B}$ cells was also significantly reduced in the

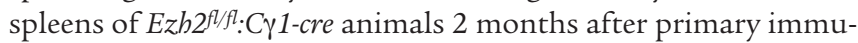
nization (Figure 2B and Supplemental Figure 2A). The reduction was already detected at the peak of the primary GC response, indicating that EZH2 is necessary for the formation of IgG1 memory B cells (Supplemental Figure 2B).

To study the role of EZH2 in antibody affinity maturation, GC $B$ cells from the spleens of NP-CGG-immunized $E z h 2^{f l / f l}: C \gamma 1$-cre: R26-yfp mice were sorted on the basis of YFP expression to enrich for Ezh2 mutants. Mutation analysis of the $V_{H} 186.2$ gene expressed by NP-specific B cells revealed a substantial reduction in the overall mutation frequency in Ezh2 mutant GC B cells (Table 1). Moreover, the frequency of replacement mutations, causing a tryptophan-to-leucine substitution at codon 33 that confers a 10-fold higher affinity for NP to the BCR, was reduced in mutant GC

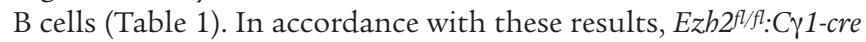
mice showed a reduction in high-affinity NP-specific IgG1 serum titers in primary and recall immune responses (Figure 2C). To assess whether the reduction in IgG1 titers was due to a intrinsic defect in Ig CSR, primary B cells from $E z h 2^{f l f l}: C r 2$-cre mice were stimulated in vitro with LPS and IL-4. Flow cytometry analysis revealed a sizeable fraction of $\mathrm{IgG} 1^{+} \mathrm{B}$ cells in Ezh2 mutant cultures (Figure 2D), thus excluding major defects in Ig CSR. The inactivation of Ezh2 in B cells was confirmed by expression of a truncated, catalytic-inert (EZH $\left.2^{\triangle S E T}\right)$ form of the protein and by a reduction in global H3K27me3 levels (Supplemental Figure 2C).

Increased apoptosis of Ezh2 mutant GC B cells is independent of $19^{A R F}$ and resistant to $B C L 2$ overexpression. To understand the mechanisms responsible for the loss of Ezh2 mutant GC B cells, Ezh2flffl: C 1 1-cre and control mice were immunized with NP-CGG and GC $\mathrm{B}$ cells were analyzed by flow cytometry for the expression of active caspases as a readout of apoptosis. Inactivation of Ezh2 led to a 3 -fold increase in the percentage of CaspGLOW ${ }^{+}$apoptotic GC B cells (Figure 3, A and B). Similar results were observed analyzing GC responses in Ezh2flfl:Cr2-cre mice (Supplemental Figure 3A). 
A
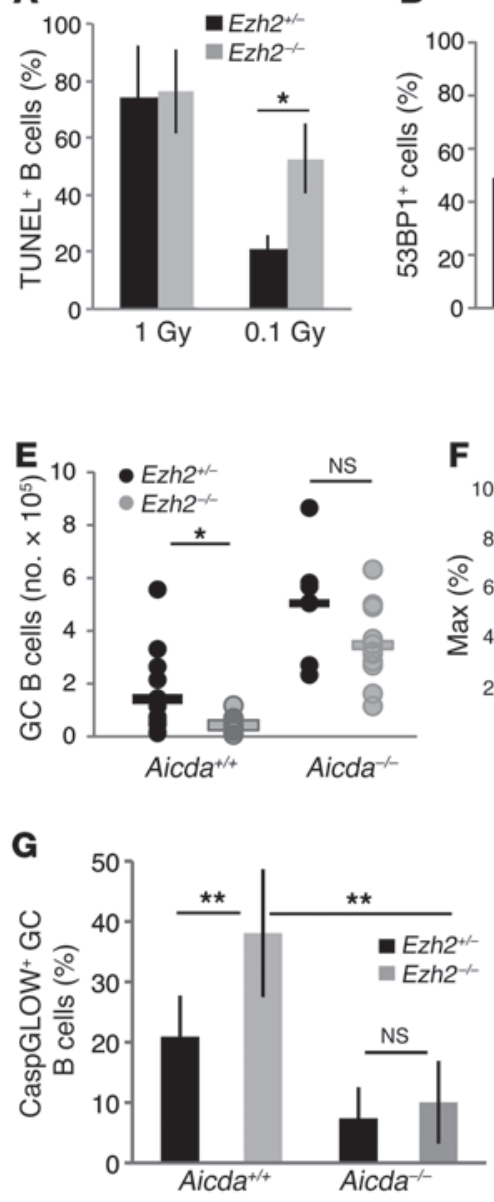

B

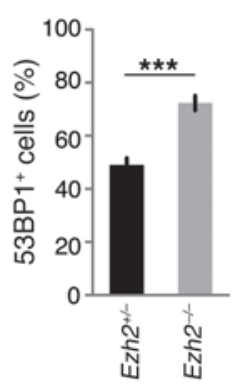

C

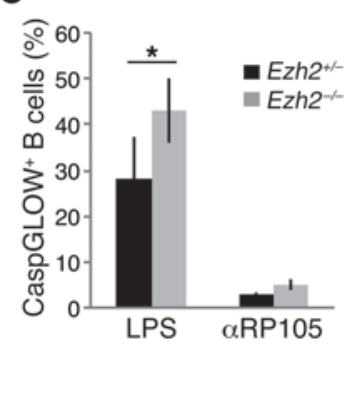

D

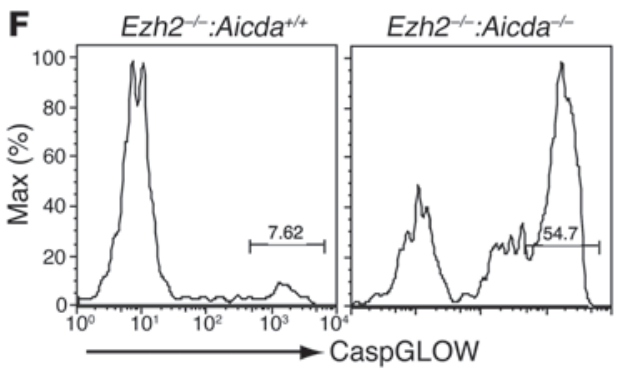

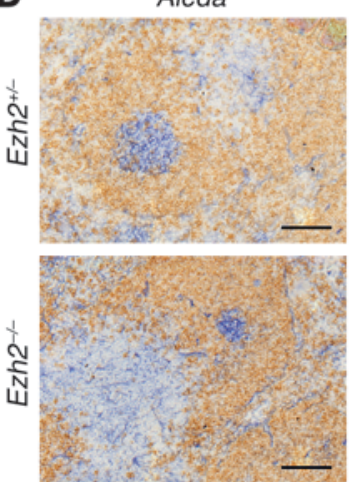

$\mathrm{Aicda}^{+/+}$
Aicda $^{-1}$

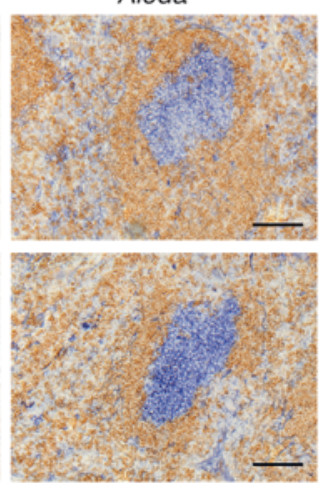

\section{Figure 4}

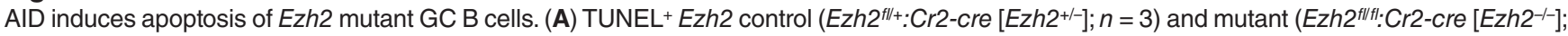

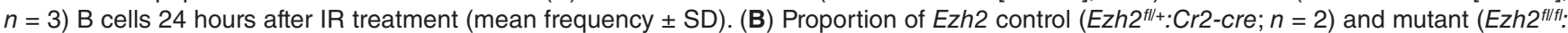
Cr2-cre; $n=2$ ) B cells with 53BP1+ foci after exposure to IR (average frequency \pm SEM). (C) Mean frequency \pm SD of CaspGLOW ${ }^{+} E z h 2$ control $\left(E z h 2^{f l++}: C r 2-c r e ; n=5\right)$ and mutant (Ezh2 $\left.{ }^{f / f f l: C r 2-c r e ; ~} n=7\right)$ B cells 3 days after LPS or $\alpha$ RP105 stimulation. (D) Histological analysis of PNA ${ }^{+}$GC (blue) within IgD+ B cell follicles (brown) in the spleen of Ezh2 control (Ezh2 fl/fl:Cr2-cre) and mutant (Ezh2 fl/fl:Cr2-cre) mice. Scale bar: $100 \mu \mathrm{m}$.

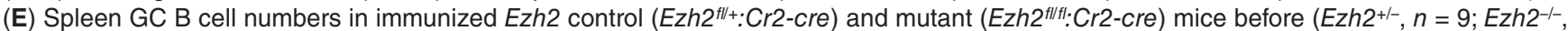
$n=6)$ and after (Ezh2 ${ }^{+/-}, n=8$; Ezh2 $\left.{ }^{-/}, n=13\right)$ Aicda inactivation. Symbols represent individual mice. (F) Representative FACS analysis of CaspGLOW+ GC B cells in Ezh2 mutant mice (Ezh2 ${ }^{\text {flfll: } C r 2-c r e) ~ b e f o r e ~ a n d ~ a f t e r ~ A I D ~ i n a c t i v a t i o n . ~(G) ~ P r o p o r t i o n ~ o f ~ s p l e n i c ~ C a s p G L O W+~ G C ~ B ~}$

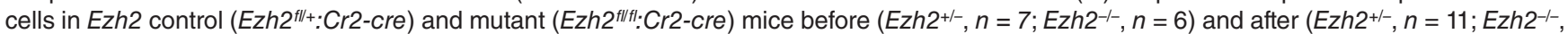
$n=13$ ) Aicda inactivation (mean \pm SD). (H) Efficiency of Ezh2 inactivation in Ezh2 mutant (Ezh2 fllf: Cr2-cre) follicular and GC B cells before $(n=1)$ or after $(n=3)$ Aicda inactivation. Columns indicate mean values. Data represent $(\mathbf{E}-\mathbf{G}) 6$, (H) $5,(\mathbf{C}) 3$, and $(\mathbf{A}, \mathbf{B}$, and $\mathbf{D}) 2$ experiments. ${ }^{\star} P<0.05 ;{ }^{* \star} P<0.01 ;{ }^{\star \star \star} P<0.0001$.

In agreement with the established role of EZH2 in the repression of the Cdkn2a tumor suppressor locus (14), Cdkn2a transcripts increased over 9 fold in Ezh2 mutant GC B cells (Figure 3C). To test whether the proapoptotic factor p19ARF, encoded by Cdkn2a, was responsible for the death of Ezh2 mutant GC B cells, we analyzed Ezh2Cdkn2a double-mutant mice (Ezh2 $2^{f / f l}: C d k n 2 a^{-/-}: C \gamma 1-c r e$ mice). Flow cytometry analysis of NP-CGG-immunized mutants and controls (Ezh2 $2^{f /+}: C d k n 2 a^{-/-}: C \gamma 1$-cre mice) revealed a significant reduction in the percentage of double-mutant GC B cells compared with that in controls (Figure 3D), which was associated with an increased percentage of apoptotic cells (Figure 3E). Moreover, genomic qPCR analysis indicated the persisting counter selection of GC B cells carrying Ezh2 recombined alleles in compound mutants (Figure 3F). Next, we attempted to rescue apoptosis of Ezh2 mutant GC B cells through enforced expression of the antiapoptotic protein BCL2, using the E $\mu B C L 2$ transgene (15). Flow cytometry analysis and quantitative assessment of the $E z h 2^{f l}$ allele revealed lower frequency of GC B cells, increased percentage of apoptotic cells, and counter selection of Ezh2 mutant GC B cells in compound mutants (Supplemental Figure 3, B-D). In conclusion, EZH2 sustains GC B cell survival independently of its repression of $p 19^{A R F}$ and without limiting mitochondrial apoptosis.

Ezh2 protects GC B cells against AID-dependent genotoxic damage. Both in vitro and in vivo studies have provided evidence that PcG proteins, including EZH2, can participate in DNA damage responses (16-19). We thus investigated whether EZH2 protected 
A

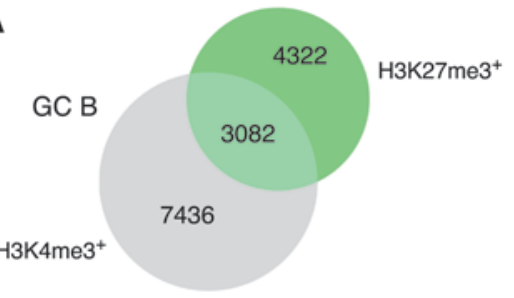

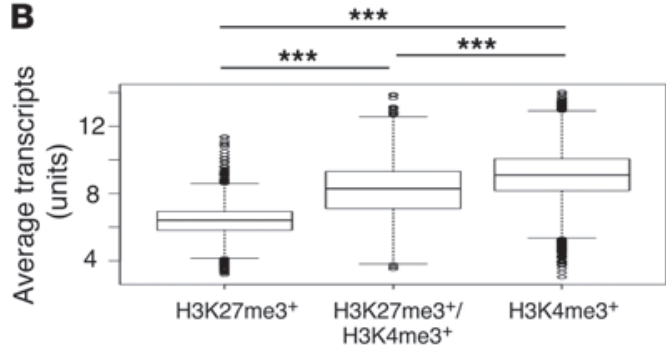

C
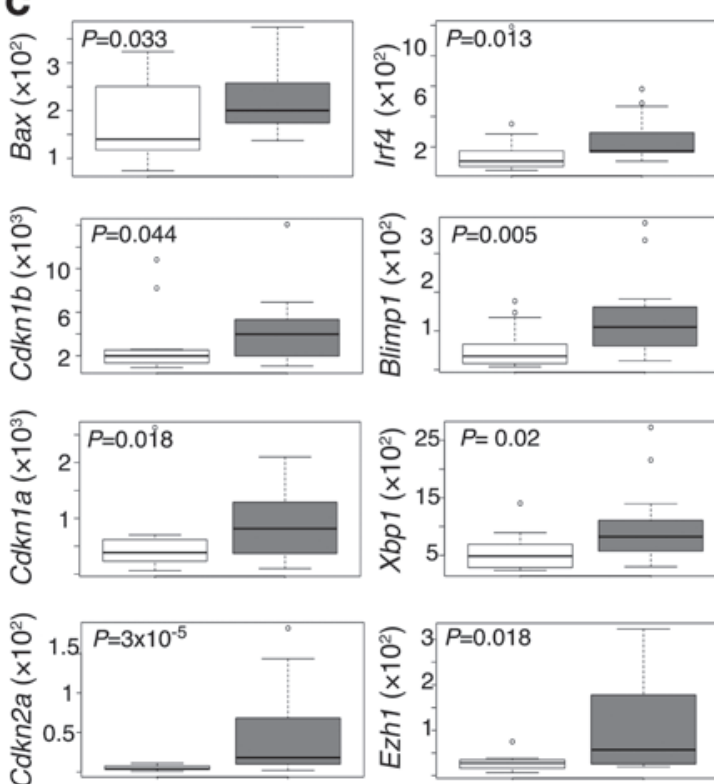
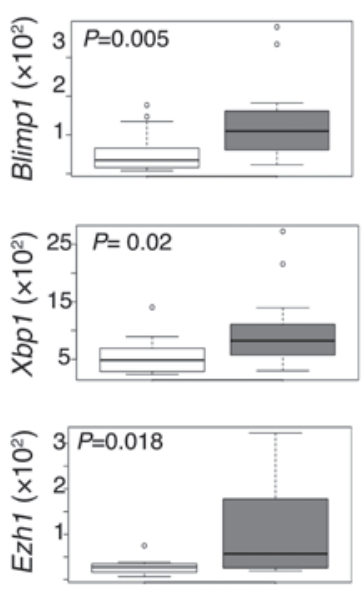
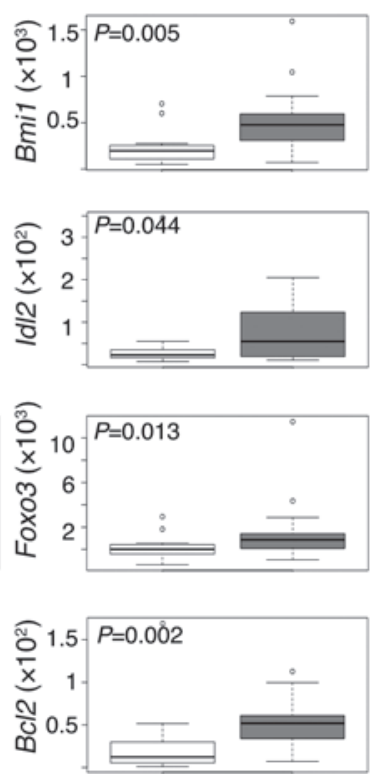

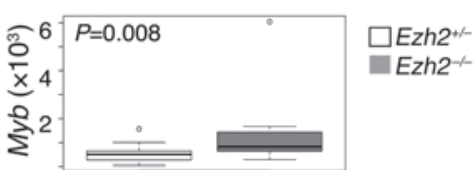

D
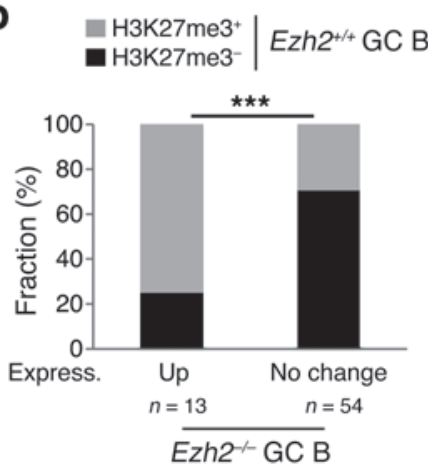

Figure 5

Genome-wide identification and characterization of H3K27me3 and H3K4me3 target genes in mouse GC B cells. (A) Venn diagram showing sets of genes marked by H3K27me3 and/or H3K4me3, respectively, in primary GC B cells purified from the spleens of immunized C57BL/6J wild-type mice. (B) Box plot of average mRNA levels of genes marked by H3K27me3 $(n=4,549)$, H3K4me3 ( $n=6,311)$, or both histone marks $(n=1,747)$ in GC B cells. ${ }^{* \star *} P<2.2 \times 10^{-16}$ ( $t$ test). (C) Box plot analysis of transcript levels of candidate genes (relative to Rplp0) quantified in Ezh2 control (Ezh2 ${ }^{f /+}: C \gamma 1$-cre [Ezh2+/-]; $\left.n=15\right)$ and mutant $\left(E z h 2^{f / / f l}: C \gamma 1\right.$-cre [Ezh2 $\left.\left.{ }^{-/}\right] ; n=17\right)$ primary GC B cells. $P$ values are indicated within each plot (Wilcoxon rank-sum test). (B and $\mathbf{C}$ ) In box-and-whisker plots, horizontal bars indicate the medians, boxes indicate 25th to 75 th percentiles, and whiskers indicate one and half time the value of the interquartile range added to the 75th percentile, or subtracted to the 25 th percentile, respectively. (D) H3K27me3 status in wild-type GC B cells of 67 candidate genes (listed in Supplemental Table 2) tested by qRT-PCR in Ezh2 control and mutant GC B cells described in C. The genes were grouped based on whether expression was upregulated (up) or remained unchanged (no change) in Ezh2 mutant GC B cells. ${ }^{* * *} P=0.0009$ (Fisher's exact test).

B cells from genotoxic damage through $\mathrm{H} 3 \mathrm{~K} 27$ trimethylation. First, we measured the response of Ezh2 mutant B cells to acute DNA damage induced by ionizing radiation (IR). Primary resting B cells purified from mutant $\left(E z h 2^{f l / f l}: \mathrm{Cr} 2\right.$-cre $)$ and control $\left(E z h 2^{f l /+}\right.$ : Cr2-cre) mice were stimulated in vitro with LPS and, shortly after, were exposed to a single dose of 0.1 and 1 Gy of IR, respectively. The fraction of apoptotic B cells was determined 24 hours after irradiation by TUNEL staining. Whereas the majority of B cells succumbed to 1 Gy of IR (irrespective of Ezh2 genotype), the treatment with 0.1 Gy caused minor toxicity to $E z h 2$ proficient B cells. In contrast, the cultures of Ezh2 mutant B cells exposed to the same sublethal IR dose consisted of mostly TUNEL ${ }^{+}$cells (Figure $4 \mathrm{~A}$ ). In accordance with this result, we found a significantly higher number of DNA damage response foci marked by 53BP1 in Ezh2 mutant B cells after exposure to IR (Figure 4B). Since AID is a major determinant of genotoxic stress in GC B cells (20), we tested whether EZH2 inhibition increased the sensitivity of B cells to AID toxicity. Initially, we compared the behavior of Ezh2 mutant $B$ cells after in vitro stimulation with either LPS/IL-4 or an antibody activating the Toll-like receptor homolog RP-105, as they differ in the ability to induce AID (Supplemental Figure 4A). Ezh2 mutant B cells responded to both mitogenic stimuli (Supplemental Figure 4B). However, only LPS/IL-4 stimulation, which induced robust AID expression, caused a significant increase in apoptosis of Ezh2 mutant B cells and lower numbers of viable cells after 4 days of in vitro culture (Figure 4C and Supplemental Figure 4C). This effect was particularly evident among IgG1 class-switched B cells (Supplemental Figure 4D). To directly assess the contribution of AID to the apoptosis of Ezh2 mutant GC B cells, Ezh2fl/f: Cr2-cre mice were crossed with AID-deficient animals. Strikingly, the size of GCs and the absolute number and the percentage of GC B cells were largely rescued in Ezh2/Aicda double mutants (Figure 4, 
A
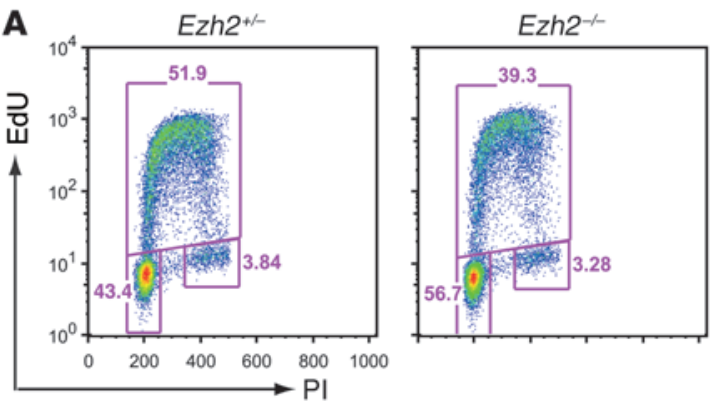

C

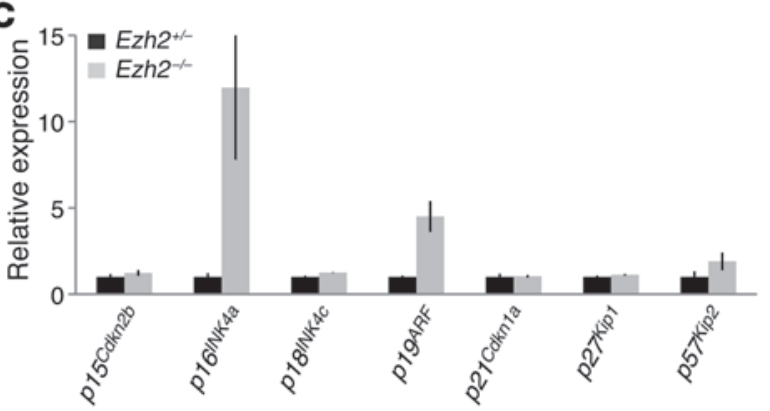

B

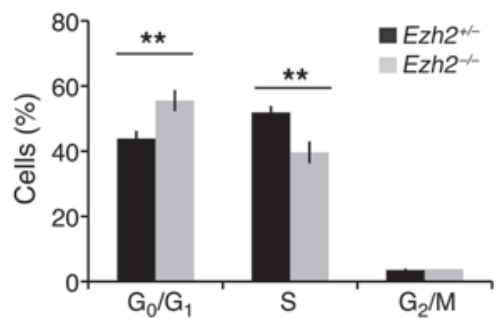

D

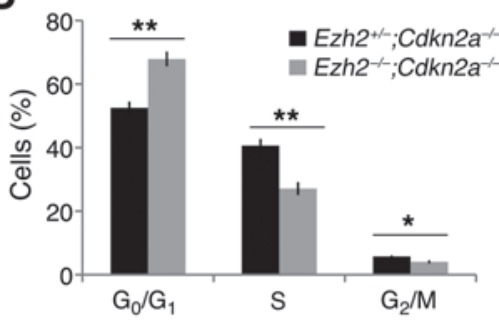

\section{Figure 6}

EZH2 regulates $\mathrm{G}_{1}$-to-S transition in $\mathrm{B}$ cells in a p16 ${ }^{\mathrm{INK} 4 \mathrm{~A}}$-independent fashion. (A) Representative cell cycle analysis of day 4 Ezh2 control $\left(E z h 2^{\mathrm{fll}+}: C \gamma 1\right.$-cre $\left.\left[E z h 2^{+-}\right] ; n=3\right)$ and mutant $\left(E z h 2^{f / f l l}: C \gamma 1\right.$-cre [Ezh2-/-]; $\left.n=4\right)$ iGB cells stained with propidium iodide $(\mathrm{PI})$ and pulsed with EdU 1 hour prior to analysis. Numbers indicate percentage of cells in $G_{0} / G_{1}$ (bottom left), $S$ (top), and $G_{2} / M$ (bottom right) phase of the cell cycle,

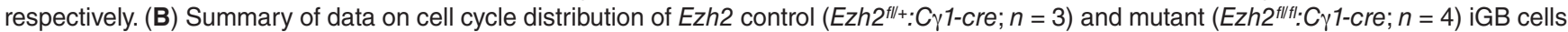
at day 4 of stimulation. (C) Transcript levels of cyclin-dependent kinase inhibitors in Ezh2 mutant (Ezh2 ${ }^{\text {fllfl: }: C \gamma 1-c r e)}$ iGB cells relative to control (Ezh2 ${ }^{\mathrm{fl}+}:$ :C 1 1-cre) and Rp/p0. Expression of $p 19 \mathrm{ARF}$ encoded by Cdkn2a was also assessed. Columns represent mean \pm SEM. (D) Summary data on cell cycle distribution of $C k n 2 a-d e f i c i e n t ~ E z h 2$ control $\left(E z h 2^{f / l+}: C \gamma 1-c r e ; n=3\right)$ and mutant $\left(E z h 2^{\text {fl/fll:}} \cdot C \gamma 1\right.$-cre; $\left.n=3\right)$ iGB cells at day 4 of stimulation. (B and D) Columns indicate mean \pm SD. ${ }^{\star} P=0.009 ;{ }^{\star \star} P=0.001$ ( $t$ test).

D and E, and Supplemental Figure 4, E and F). Importantly, AID inactivation led to the normalization of apoptosis of Ezh2 mutant GC B cells (Figure 4, F and G), allowing for the first time efficient recovery of cells that had recombined both $E z h 2^{f l}$ alleles (Figure $4 \mathrm{H})$. These results show that EZH2 protects against DNA damage-induced apoptosis, in particular, in GC B cells, genotoxicity linked to AID mutagenesis.

Ezh2 regulates the GC B cell transcriptional program. Despite the consistent normalization of apoptosis, Ezh2 fl/fl:Aicda-/-:Cr2-cre compound mutants showed still a modest, yet reproducible, reduction in GC B cell numbers and, in few cases, Ezh2/Aicda double-mutant GC B cells remained counter selected (Supplemental Figure 4G). These results suggested additional function(s) of Ezh2 in GC B cells. To address this, we performed H3K27me3 ChIP assays coupled to high-throughput sequencing (ChIP-seq) to identify Ezh2 target genes in GC B cells at a genome-wide level. We used the modelbased analysis of ChIP-seq peak calling software and applied a stringent $P$ value $\left(P<10 \times 10^{-10}\right)$ cutoff for peak detection that we recently validated to uncover the core subset of polycomb targets in somatic cell reprogramming (21). A similar approach was used for global identification of targets of $\mathrm{H} 3 \mathrm{~K} 4 \mathrm{me} 3$ that marks actively transcribed loci. In GC B cells, we identified 7,404 genes that carried $\mathrm{H} 3 \mathrm{~K} 27 \mathrm{me} 3$ in a $10-\mathrm{Kb}$ genomic interval centered on the TSS. Of these targets, $41 \%$ (3,082 out of 7,404 genes) carried the $\mathrm{H} 3 \mathrm{~K} 4 \mathrm{me} 3$ mark also, thus representing bivalent genes (Figure $5 \mathrm{~A}$ and Supplemental Table 1). Instead, 7,436 genes were exclusively marked by H3K4me3 (Figure 5A and Supplemental Table 1).
To gain insights into the biological function of $\mathrm{H} 3 \mathrm{~K} 27 \mathrm{me} 3$ targets in GC B cells, we performed a Gene Ontology analysis. Genes marked by H3K27me3 were significantly $(P \leq 0.01)$ enriched for categories involved in developmental processes (Supplemental Figure 5A), whereas $\mathrm{H} 3 \mathrm{~K} 4 \mathrm{me} 3$ preferentially marked genes involved in metabolism (Supplemental Figure 5B). To understand the influence of H3K27me3 on target gene expression, we compared the average transcript levels of genes marked in GC B cells, by $\mathrm{H} 3 \mathrm{~K} 27 \mathrm{me} 3, \mathrm{H} 3 \mathrm{~K} 4 \mathrm{me} 3$, or by both, respectively. In accordance with previous observations (22), we found that genes marked by H3K27me3 were significantly $\left(P<2.2 \times 10^{-16}\right)$ less transcribed than those marked by H3K4me3 (Figure $5 \mathrm{~B}$ ). Transcripts encoded by bivalent genes showed intermediate levels, which were significantly lower $\left(P<2.2 \times 10^{-16}\right)$ than those derived from H3K4me3-marked genes (Figure 5B). These data pointed to a role for EZH2 in transcriptional silencing of target genes in GC B cells. To test this, we quantified the transcript levels of a representative set of genes in Ezh2 mutant and control GC B cells $(n=67)$ selected according to H3K27me3 status and relevance in B cell biology (Supplemental Table 2). We found that differentially expressed genes $(P<0.05)$ were all upregulated in Ezh2 mutant GC B cells (Figure 5C) and preferentially marked by H3K27me3 in Ezh2-proficient GC B cells (Figure $5 \mathrm{D}$ and Supplemental Figure 5C). These included key regulators of cell cycle progression (Cdkn1a, Cdkn1b, and Cdkn2a) and GC B cell differentiation (Id2, Blimp1, Irf4, and Xbp1; Figure 5C). For IRF4, we confirmed the upregulation at the protein level. Moreover, the fraction of cells expressing high IRF4 levels increased significantly 
A
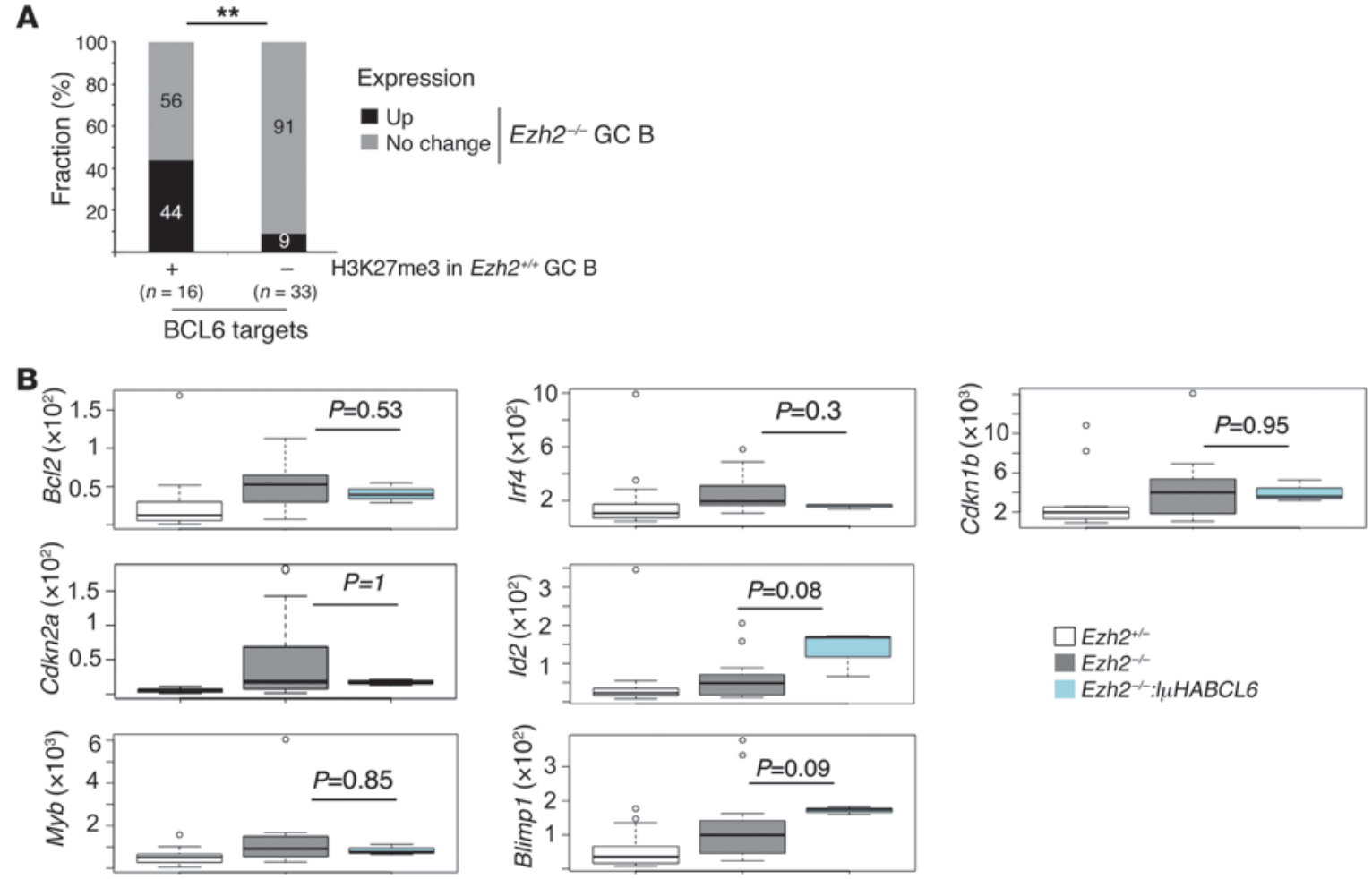

\section{Figure 7}

EZH2 modulates BCL6 repression in GC B cells. (A) Percentage of a representative set of BCL6 target genes (FDR $\leq 0.0001$; Supplemental Table 3 ) that are significantly $(P<0.05)$ upregulated in Ezh2 mutant GC B cells, classified on the basis of H3K27me3 status in their wild-type counterparts. ${ }^{* \star} P=0.004$ (Fisher's exact test). (B) Box plot representation of transcript levels (relative to Rp/p0) of a representative set of BCL6 target genes

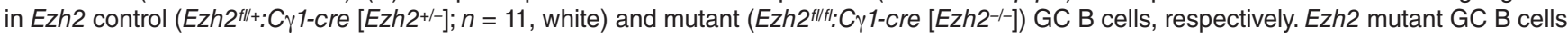
were compared before ( $n=14$, gray) and after $(n=3$, blue) complementation with the luHABCL6 transgene. Significance of the differences in gene expression levels is indicated within each plot (Wilcoxon rank-sum test). Horizontal bars indicate the medians, In box-and-whisker plots, horizontal bars indicate the medians, boxes indicate 25th to 75th percentiles, and whiskers indicate one and half time the value of the interquartile range added to the 75 th percentile, or subtracted to the 25 th percentile, respectively.

in GCs of Ezh2 conditional mutants (Supplemental Figure 6, A and $\mathrm{B}$ ). Instead, the expression of $\mathrm{H} 3 \mathrm{~K} 4 \mathrm{me} 3$-only targets remained largely unaffected in response to Ezh2 inactivation (data not shown). Collectively, these data assign a critical control over cell cycle progression and differentiation of GC B cells to EZH2.

Ezh2 facilitates B cell proliferation independent of repression of CDK inhibitors. The identification of Cdkn1a, Cdkn1b, Cdkn2a as targets of $\mathrm{H} 3 \mathrm{~K} 27 \mathrm{me} 3$ in GC B cells pointed to a role for EZH2 in the regulation of cell cycle progression, possibly through the repression of CDK inhibitors $(8,23)$. To test this hypothesis, given the difficulty in tracking the few Ezh2 mutant GC B cells in vivo, we performed cell cycle distribution analysis after in vitro activation of Ezh2 mutant B cells with membrane-bound CD40L and IL-4. Acute EZH2 inactivation in B cells led to an accumulation of cells in the $G_{0} / G_{1}$ phase of the cell cycle (Figure 6, A and B). Expression analysis revealed a substantial upregulation of the CDK inhibitor p16 ${ }^{\text {Ink4a }}$ in Ezh2 mutant B cells (Figure 6C). To determine whether induction of $\mathrm{p} 16^{\text {Ink4a }}$ was responsible for the defect in $\mathrm{G}_{1}$-to-S transition of Ezh2 mutant B cells, we analyzed the cell cycle profile of Ezh2/Cdkn2a double-mutant B cells after CD40L/IL-4 stimulation. In contrast to previous results $(24,25), C d k n 2 a$ inactivation failed to normalize the cell cycle profile of $E z h 2$ mutant B cells (Figure 6D). Moreover, expression of other CDK inhibitors was largely unaffected in Ezh2 mutant primary B cells after mitogenic stimulation, excluding their possible involvement in the cell cycle defect (Figure 6C). All together these results unravel a novel mechanism that uncouples EZH2 control of $\mathrm{G}_{1}$-to-S transition from the repression of CDK inhibitors.

Ezh2 is required for repression of a subset of BCL6 targets in GC B cells. We noticed that several genes upregulated in Ezh2 mutant GC $B$ cells are bound with high significance and repressed by the transcriptional repressor BCL6 in GC B cells (26). Given the relevance of BCL6 in the regulation of the GC reaction (27), we investigated the influence of EZH2 on BCL6-dependent gene silencing. Specifically, we quantified the transcript levels of 49 highly significant (false discovery rate $[\mathrm{FDR}] \leq 0.0001$ ) BCL6 targets in Ezh2 mutant GC B cells (27). Of these, around one-third $(n=16)$ were marked by H3K27me3 in wild-type GC B cells (Supplemental Table 3). Whereas expression of BCL 6 targets lacking H3K27me3 remained largely unaltered by Ezh2 inactivation (3 out of 33 genes), nearly half of the genes bound by PRC 2 and BCL6 in wild-type GC B cells (7 out of 16 genes; Bcl2, Id2, Cdkn1b, Cdkn2a, Myb, Blimp1, and Irf4) were upregulated in the Ezh2 mutant counterparts (Figure 5C, Figure 7A, and Supplemental Table 3). To exclude that EZH2 regulated the $B C L 6$ gene, we crossed conditional Ezh2

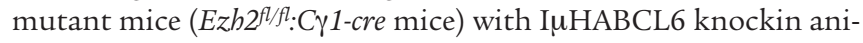


A

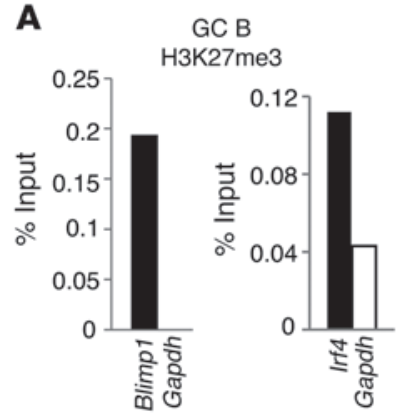

D

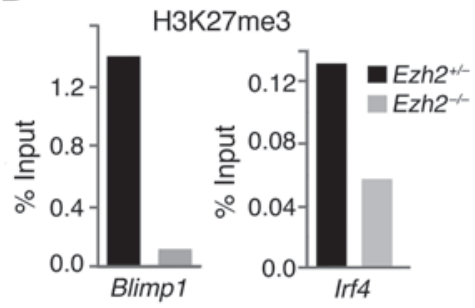

B

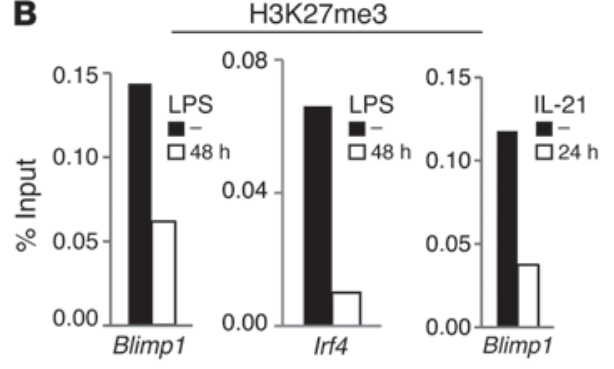

C

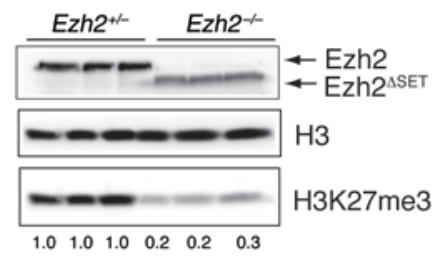

E

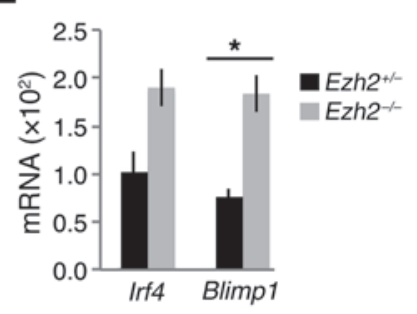

F

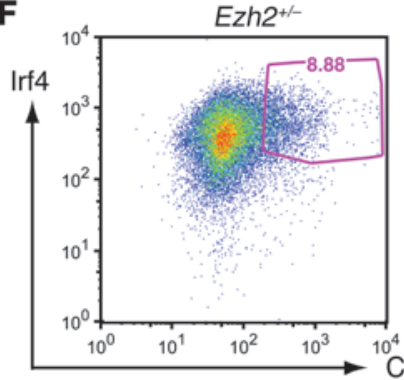

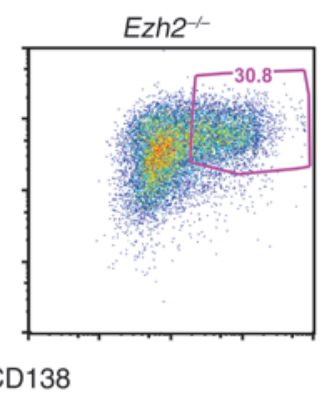

G

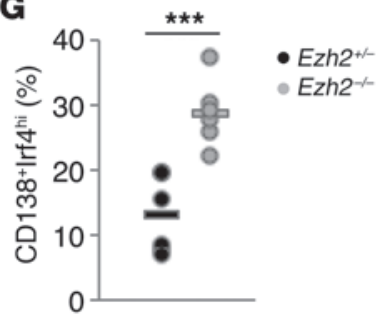

H

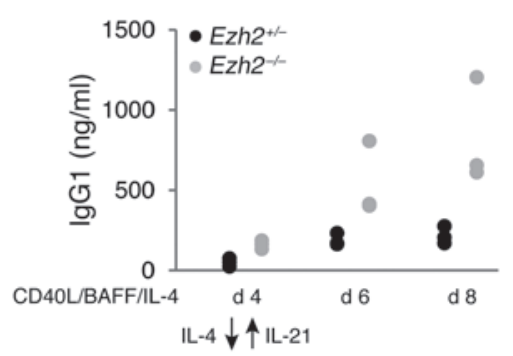

\section{Figure 8}

H3K27me3 marks Blimp1 and Irf4 in GC B cells and restricts PC differentiation. (A) Blimp1 and Irf4 H3K27me3 ChIP-qPCR in primary GC $\mathrm{B}$ cells. Gapdh was assessed as negative control. Enrichment is relative to input and histone $\mathrm{H} 3$ density. Columns represent mean values. (B) Blimp1 and Irf4 H3K27me3 ChIP-qPCR in I.29 $\mu^{+}$B cells before (-) and 48 hours after LPS stimulation and in A20 cells in response to 24 hours of IL-21 stimulation. (C) Immunoblot analysis of H3K27me3 and full-length (Ezh2)/truncated (Ezh2SET) EZH2 levels in representative Ezh2 control

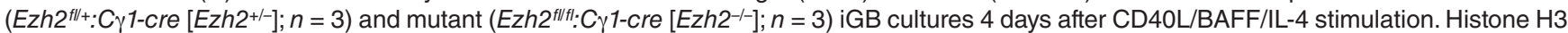
was assessed as loading control. Quantification of H3K27me3 levels relative to controls is shown. (D) Blimp1 and Irf4 H3K27me3 ChIP-qPCR

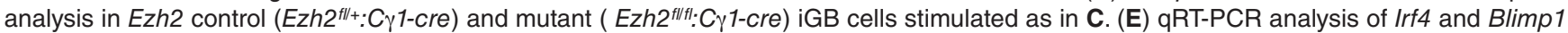

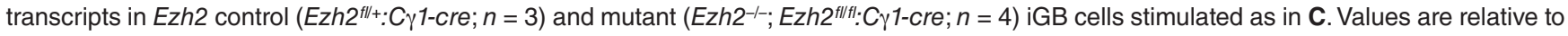
Rplp0. Columns indicate mean \pm SEM. ${ }^{*} P=0.006$ ( $t$ test). (F) Representative FACS analysis and (G) frequency of Irf4hiCD138+ PCs in control

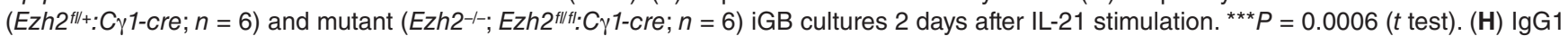

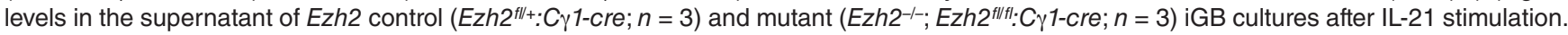
$(\mathbf{G}$ and $\mathbf{H})$ Symbols represent individual samples; bars refer to mean values. (B and $\mathbf{D})$ Fold enrichments are calculated as in $\mathbf{A}$.

mals expressing BCL 6 under the I $\mu$ promoter that is constitutively active in B cells (28). Expression analysis performed on primary GC $B$ cells of compound mutants revealed the failure of I $\mu$ HABCL6 to normalize expression of a subset of H3K27me3/BCL6 shared targets (Figure 7B). These results indicate that $\mathrm{EZH} 2$ regulates BCL6 repressor function on a subset of common targets in GC B cells.

H3K27me 3 marking of Blimp1 and Irf4 in GC B cells is erased upon induction of PC differentiation. Bioinformatic analysis of ChIPseq data identified the PC determinant Blimp1 as a target of $\mathrm{H} 3 \mathrm{~K} 27 \mathrm{me} 3$ in mouse GC B cells, in accordance with recent findings in the human counterparts (23). We confirmed this result by performing $\mathrm{H} 3 \mathrm{~K} 27 \mathrm{me} 3 \mathrm{ChIP}-\mathrm{qPCR}$ analyses on primary GC $\mathrm{B}$ cells (Figure $8 \mathrm{~A}$ ) and A20 and I.29 $\mu^{+}$murine B lymphoma cells (Supplemental Figure 7A) expressing low levels of Blimp1 (Sup- plemental Figure 7F). Accordingly, the PRC2 core component Suz12 was recruited to the Blimp1 locus in BLIMP-negative B cells (Supplemental Figure 7B). In a similar fashion, screening by ChIPqPCR identified $\mathrm{H} 3 \mathrm{~K} 27 \mathrm{me} 3$ deposition in a region proximal to the Irf4 TSS in primary GC B cells and A20 and I. $29 \mu^{+}$lymphoma cells (Figure 8A and Supplemental Figure 7A). These results establish that Blimp1 and Irf4 are direct targets of PRC2 in GC B cells.

To study the regulation of $\mathrm{H} 3 \mathrm{~K} 27$ trimethylation at the Blimp1 and Irf4 loci during terminal differentiation, we stimulated B cells in vitro with either LPS or IL-21. A substantial loss of H3K27me3 at the Blimp1 and Irf4 loci (Figure 8B) was detected within the first 24-48 hours after stimulation. In particular, for Irf4, this coincided with loss of Suz12 binding, increased H3K4 trimethylation, and recruitment of RNA polymerase II to the promoter, which correlated 
A

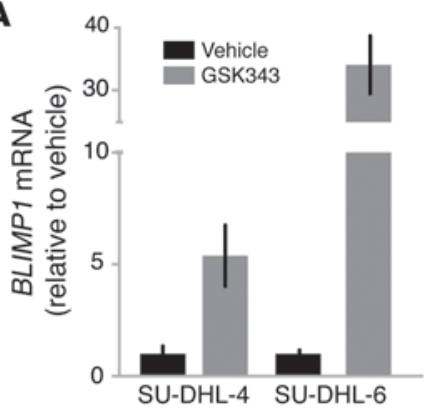

C

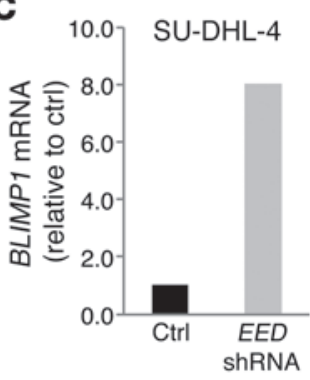

E

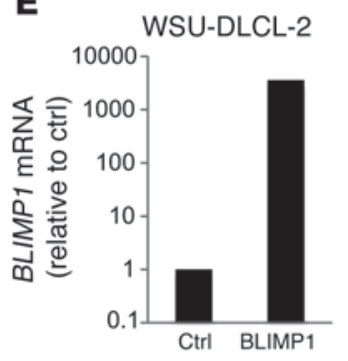

B
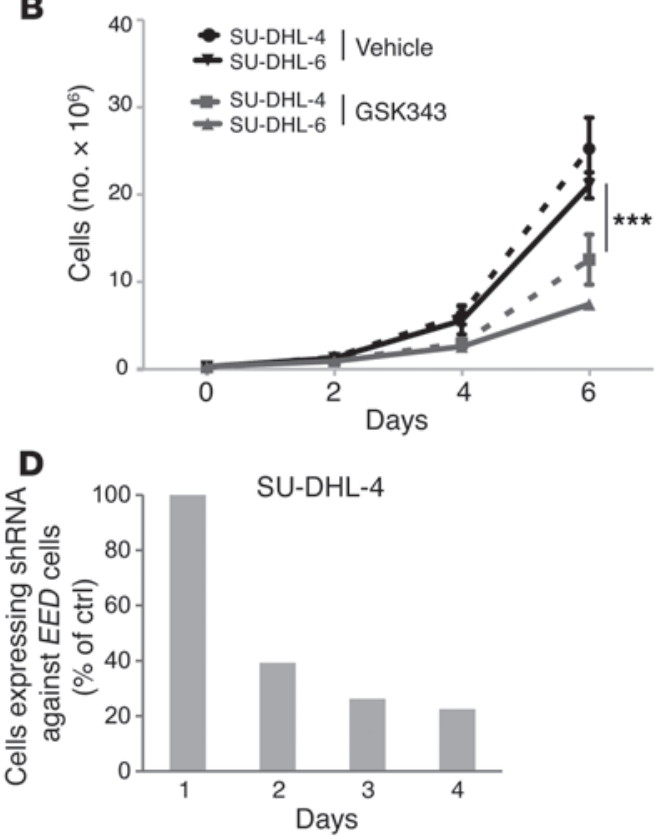

$\mathbf{F}$

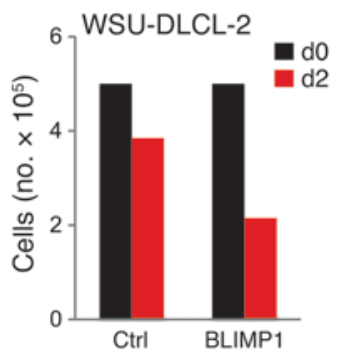

Figure 9

Y641 mutant of EZH2 silences BLIMP1 expression through PRC2 to facilitate DLBCL growth. (A) BLIMP1 transcripts in SU-DHL-4 and SUDHL-6 DLBCL cells 12 days after treatment with GSK343 or vehicle, as revealed by qRT$\mathrm{PCR}$ analysis. Columns indicate mean $\pm \mathrm{SD}$ of triplicates. Expression is relative to that of vehicle-treated control cells after normalization for RPLPO expression. (B) Growth curves of SU-DHL-4 (black line) and SU-DHL-6 (gray line) DLBCL cells treated with either GSK343 (solid line) or vehicle (dotted line). Average cell numbers at the indicated time points \pm SEM of 4 replicates is shown. ${ }^{* * *} P<0.001$ (2-way ANOVA). (C) BLIMP1 transcript levels in SU-DHL-4 DLBCL cells after infection with lentiviruses expressing control (ctrl) or anti-EED (EED) shRNAs, respectively. Results are presented relative to control after normalization for GAPDH expression. Columns indicate mean of 2 independent experiments. (D) Frequency of SUDHL-4 DLBCL cells expressing shRNA against EED relative to those expressing control shRNA at the indicated days after lentivirus infection. (E) qRT-PCR analysis of Blimp1 transcript levels in WSU-DLCL-2 DLBCL cells transfected with control or BLIMP1 expression vector, respectively. Columns indicate mean value of 2 independent experiments. Values are presented relative to control after normalization for HPRT expression. (F) Comparison of WSU-DLCL-2 cell numbers at the indicated days after transfection with control or Blimp1 expression vector, respectively. Data are representative of 2 experiments. with augmented Irf4 transcription (Supplemental Figure 7, C-E). Notably, induction of PC differentiation did not promote global H3K27 demethylation, as PRC2 targets Hoxa9 and Olig1 remained marked by H3K27me3 and bound by Suz12 in stimulated B cells (Supplemental Figure 7G). These results reveal that Blimp1 and Irf4 upregulation during onset of $\mathrm{PC}$ differentiation is accompanied by a substantial loss of $\mathrm{H} 3 \mathrm{~K} 27 \mathrm{me} 3$ proximal to their TSS.

Ezh2 restricts terminal B cell differentiation promoted by IL-21. To assess the relevance of EZH2-dependent repression of Blimp1 and Irf4 in GC B cells, we determined the effects of acute Ezh2 inactivation on terminal differentiation. Primary Ezh $2^{f l f l}: C \gamma 1$-cre B cells were stimulated in vitro for 4 days with CD40L, BAFF, and IL-4 to generate proliferating GC-like B cells (also called iGB cells; ref. 29) that were ultimately differentiated into PCs by the addition of IL-21 to the culture media (29). Efficient Ezh2 inactivation in iGB cells led to a substantial reduction in global H3K27me3 levels (Figure 8C) that was confirmed at the Blimp1 and Irf4 loci by ChIP-qPCR analysis (Figure 8D). Ezh2 mutant iGB cells expressed higher Irf4 and Blimp1 transcript levels (Figure 8E), which was alone sufficient to trigger differentiation of a subset of CD40-activated B cells into Irf4hiCD $138^{+}$PCs (Supplemental Figure 8A). Importantly, upon IL-21 stimulation, over one-third of Ezh2 mutant iGB cells differentiated into Irf4 ${ }^{\text {hi }} \mathrm{CD} 138^{+}$PCs, significantly outnumbering those present in control cultures (Figure 8, F and G). In accordance with this result, conditioned media of Ezh2 mutant iGB cultures stimulated with IL-21 contained higher levels of soluble IgG1 antibodies
(Figure $8 \mathrm{H}$ ), which coincided with a greater number of $\operatorname{IgG1} 1^{+}$antibody-secreting cells, when compared with controls (Supplemental Figure $8 \mathrm{~B}$ ). These results indicate that EZH2 repression of Blimp1 and Irf4 restricts IL-21-controlled PC differentiation.

Y641 mutant of EZH2 enforces BLIMP1 repression to support DLBCL growth. BLIMP1 has been proposed tumor suppressor since its inactivation occurs frequently in human DLBCL and promotes B cell lymphomas in mouse models (30-32). In DLBCL, impaired BLIMP1 expression results from genetic alterations of the BLIMP1 locus or stable repression by BCL6 (32). BLIMP1 mutations have been almost exclusively associated with $A B C$-type DLBCL $(30,32)$. Since $E Z H 2$ gain-of-function mutations occur predominantly in GC-type DLBCL (9) and given that Blimp1 is a direct target of EZH2 repression in primary mouse GC B cells, we investigated whether EZH2 was required for stable BLIMP1 silencing in GC-type DLBCL. To this aim, we treated the DLBCL cells lines, SU-DHL-4 and SU-DHL-6, expressing constitutively active forms of EZH2 $(9,33)$ with the EZH2 small molecule inhibitor, GSK343 (34). Treatment of tumor cells with $2.5 \mu \mathrm{M}$ GSK343 led to a reduction in global levels of H3K27me3, without interfering with EZH2 expression (Supplemental Figure 9, A and B). Importantly, exposure of DLBCL cells to GSK343 induced a substantial upregulation of BLIMP1 expression in both DLBCL lines (Figure 9A). This effect was associated with impaired in vitro lymphoma growth (Figure 9B) that was contributed by a partial arrest in cell cycle progression at the $\mathrm{G}_{1}$-to-S 
transition (Supplemental Figure 9C). To understand whether Y641 mutant of EZH2 exerted its catalytic activity within PRC2, we inhibited the expression of the essential PRC2 subunit EED in DLBCL cells. Downregulation of EED in SU-DHL-4 cells (Supplemental Figure 9, D and E) caused a global reduction in H3K27me3 levels (Supplemental Figure 9F) and, most importantly, a substantial upregulation of BLIMP1 expression (Figure 9C). Similar results were obtained in A20 lymphoma B cells expressing wild-type EZH2 (Supplemental Figure 10). Inhibition of EED interfered with in vitro DLBCL growth (Figure 9D) and impaired cell cycle progression (Supplemental Figure $9 \mathrm{G})$, extending our data as well as that of others based on the use of EZH2 small molecule inhibitors (23, 35-37). In a subset of SU-DHL-4 cells, PRC2 inhibition alone was sufficient to cause the induction of surface CD138 expression and the downregulation of MHC class II molecules, which accompanies terminal differentiation (Supplemental Figure 9H). To test whether induction of BLIMP1 was alone sufficient to influence growth of B lymphoma cells expressing a constitutively active form of EZH2, we complemented WSU-DLCL-2 DLBCL cells with a BLIMP1 expression vector. Transient expression of BLIMP1 (Figure 9E) caused a severe impairment in in vitro growth of DLBCL, which ultimately led to fewer lymphoma cells retrieved 2 days after complementation (Figure 9F). All together, these results indicate that constitutively active $\mathrm{EZH} 2$ represses the tumor suppressor BLIMP1 via PRC2 to facilitate the growth of GC-type DLBCL.

\section{Discussion}

The strong induction of EZH2 in GC B cells, combined with the frequent occurrence of $\mathrm{EZH} 2$ gain-of function mutations in GCderived NHL, suggested a role for the PcG protein in the regulation of the GC reaction. Indeed, using cell type-specific gene targeting in mice, we show that Ezh2 methyltransferase activity is strictly required for GC B cell function. EZH2 inactivation resulted in fewer GCs, reduced numbers of high-affinity long-lived memory $\mathrm{B}$ cells, and impaired production of antigen-specific antibodies upon encounter with T cell-dependent antigens.

The failure to sustain the GC reaction was largely contributed by enhanced apoptosis of Ezh2 mutant B cells, which was independent of $p 19^{A R F}$ upregulation and resistant to constitutive BCL2 expression. Strikingly, instead, GC B cells lacking functional EZH2 became highly vulnerable to genotoxic stress associated with AID function. As a consequence of premature death, Ezh2 mutant GC $\mathrm{B}$ cells carried fewer Ig V gene mutations and failed to be selected by antigen-driven selection. The enrichment for apoptotic cells among Ig class-switched Ezh2 mutant B cells suggests a possible contribution of the PcG protein to the repair of DNA breaks generated during $\mathrm{IgH}$ isotype switching, extending previous findings $(16,18,19,38)$. In support of this, Ezh2 mutant primary B cells displayed higher numbers of DNA damage foci marked by 53BP1 after exposure to a sublethal dose of IR. The failure of $E z h 2$ mutant GC B cells to preserve genome integrity may trigger ultimately their death, possibly as a consequence of mitotic catastrophe. This scenario does not exclude that EZH2 may act to limit the genomewide recruitment of AID via $\mathrm{H} 3 \mathrm{~K} 27 \mathrm{me} 3$-dependent silencing of target genes $(39,40)$. Future studies will establish whether EZH2 inactivation increases the frequency of off-target mutations caused by AID, in particular at bivalent genes $(40,41)$.

Global identification of $\mathrm{H} 3 \mathrm{~K} 27 \mathrm{me} 3$ targets combined with expression analyses revealed a critical contribution of EZH2 to the regulation of the GC B cell transcriptional program. H3K27me3 targets were preferentially repressed in GC B cells and strongly enriched for developmental regulators, supporting the notion that, in differentiated cells, PRC2 has a critical function to preserve silencing of transcriptional programs driving alternative cell fates. Importantly, EZH2 also exerted a direct control of the expression of a substantial number of genes that regulate the persistence and expansion of B cells within the GC reaction. EZH2 was required to repress $I d 2$, the inhibitor of the E-box protein E2A, whose function is necessary for GC B cells (42). In GC B cells, EZH2 was also critical to prevent the expression of the cyclin-dependent kinase inhibitor $p 16^{I N K 4 A}$ encoded by the Cdkn2a locus, which regulates $\mathrm{G}_{1}$-to-S transition. In accordance with this, a higher proportion of Ezh2 mutant cells was arrested at the $\mathrm{G}_{0} / \mathrm{G}_{1}$ stage after CD40 stimulation. Surprisingly, in contrast to previous reports, the cell cycle defect was neither rescued by $C d k n 2 a$ inactivation nor associated with substantial changes in the expression of other CDK inhibitors. These results unravel a novel mechanism through which EZH2 facilitates $\mathrm{G}_{1}$-to-S progression that is independent of CDK repression.

Of note, the majority of $\mathrm{H} 3 \mathrm{~K} 27 \mathrm{me} 3$ targets that we found upregulated in Ezh2 mutant GC B cells are bound and repressed by BCL6 (26), which is essential for GC B cell function (27). This result underscores a possible cooperation between PRC2 and BCL6 in the repression of common targets, which include important negative regulators of GC B cell function, such as Id2 and Blimp1. Our data support a scenario in which EZH2 primarily regulates BCL6 repressor activity, as enforced BCL6 expression was not sufficient in Ezh2 mutant GC B cells to restore repression of a representative subset of shared targets.

One of the main findings of this study is the identification of PC determinants Blimp1 and Irf4 (43-45) as direct targets of EZH2 repression in GC B cells. Blimp1 and Irf4 genes were marked by H3K27me3 and bound by PRC2 in B cells prior to onset of PC differentiation. This regulation was critical to repress Blimp1 and Irf4, since transcripts for both PC determinants were significantly upregulated in Ezh2 mutant GC B cells. The induction of the PC program in GC B cells following EZH2 inhibition was completed by the upregulation of $X b p 1$, which is required to sustain high rates of Ig synthesis $(46,47)$. Acute Ezh2 inactivation was sufficient to trigger terminal differentiation of a subset of CD40-activated B cells. More strikingly, the addition of IL-21 substantial boosted differentiation of PcG mutant B cells into antibody-secreting cells in response to CD40 stimulation. This result indicates that EZH2 limits the amplitude of the PC response induced by IL-21 (48), thereby possibly extending the persistence of B cells in the GC $(49,50)$. We propose that, in GC B cells, EZH2 modulates IL-21dependent Blimp1 expression via H3K27 trimethylation $(51,52)$. Therefore, signals regulating PRC2 recruitment at the Blimp1 locus are predicted to influence the balance between IL-21-dependent Bcl 6 and Blimp1 expression and, thereby, the decision of B cells to persist rather than exit from the GC reaction. The control of Irf4 expression exerted through $\mathrm{H} 3 \mathrm{~K} 27 \mathrm{me} 3$ is likely to contribute to the regulation by EZH2 of the fate of GC B cells (53).

The increased susceptibility of Ezh2 mutant B cells to PC differentiation is seemingly in contrast with reduced serum Ig titers measured in PcG mutant animals after immunization with T celldependent antigens. The increased apoptosis may represent a major obstacle for Ezh2 mutant B cells to exit the GC as antibodysecreting cells. Moreover, whereas EZH2 inactivation interferes 
neither with Blimp1-controlled Xbp1 expression nor with the establishment of the transcriptional program that supports the unfolded protein response (short-lived Ezh2 mutant PCs secrete normal amounts of antibodies in vitro), additional experiments will be required to determine whether EZH2 is required for longterm PC persistence (54).

The identification of Blimp1 as a direct target of EZH2 is relevant for the understanding of the mechanisms through which the PcG protein may contribute to the pathogenesis of DLBCL. BLIMP1 loss-of-function mutations occur frequently in ABC-type DLBCL and accelerate lymphomagenesis in mouse models, supporting its role as tumor suppressor (30-32). The rare occurrence of BLIMP1 mutations in GC-type DLBCL suggests the existence of additional mechanisms that could prevent its expression in this form of NHL. Using pharmacological inhibition of EZH2 and functional inactivation of PRC2, we provide evidence that constitutively active EZH2 enforces BLIMP1 silencing in GC-type DLBCL cells. While our manuscript was under review an independent study reached similar conclusions (23). Importantly, we provide evidence that BLIMP1 induction alone is sufficient to impair in vitro growth of GC-type DLBCL expressing mutant EZH2. Thus, epigenetic silencing of BLIMP1 may represent an important mechanism through which EZH2 supports lymphoma growth.

In conclusion, our data indicate that EZH2 methyltransferase activity is strictly required for the establishment of a protective long-term B cell adaptive immune response. EZH2 accomplishes this function through the coordinated regulation of GC B cell differentiation, proliferation, and response to genotoxic damage imposed by AID. Conversely, enforced BLIMP1 repression, coupled to protection against AID mutagenesis, acceleration of S-phase entry, and support for BCL6 function, may represent the mechanism through which constitutively active EZH2 contributes to lymphomagenesis. These results provide a rationale for use of EZH2 inhibitors (35-37) in combination with inducers of terminal differentiation, such as IL-21 $(55,56)$ and genotoxic agents, in the treatment of GC-derived DLBCL and follicular lymphoma.

\section{Methods}

Mice. Ezh2fl, R26-yfp, Cr2-cre, C $11-c r e, C d k n 2 a^{-/}, E \mu-B C L 2, A i c d a^{-/}$, and $I \mu H A B C L 6$ mice were previously described (11-13, 28, 57-60). Compound mutants were backcrossed to C57BL/6J mice for 6 or more generations and kept under specific pathogen-free conditions. Eight- to sixteen-week-old mice were immunized by intraperitoneal injection of alum-precipitated NP$\mathrm{CGG}_{25-36}$ (100 $\mu \mathrm{g}$ per mouse; Biosearch Technologies) or $\mathrm{NP}-\mathrm{CGG}_{25} / \mathrm{PBS}$ (50 $\mu \mathrm{g}$ per mouse) for secondary responses. Sheep red blood cells $\left(1 \times 10^{8}\right.$ cells per mouse; Oxoid) were resuspended in PBS and injected intraperitoneally.

Antibodies. Flow cytometry was performed using fluorescent- or biotinlabeled antibodies reactive to mouse CD19 (1D3; eBioscience), CD21 (8D9; eBioscience), CD23 (B3B4; eBioscience), CD38 (90; eBioscience), IgD (11.26; eBioscience), CD184 (2B11; eBioscience), CD95 (Jo2; BD Biosciences), CD138 (281-2; BD Biosciences), IgG1 (A85-1; BD Biosciences), IgM (R33.24.12; K. Rajewsky, Max Delbrück Center for Molecular Medicine, Berlin, Germany), CD45R/B220 (RA3-6B2; K. Rajewsky), and Igא (R33-1810; K. Rajewsky). Antibodies against human MHC class II and CD138 were provided by S. Cenci (San Raffaele Scientific Institute). NP binding was assessed using NIP-BSA conjugated to allophycocyanin (provided by M. Hikida, Kyoto University, Kyoto, Japan and T. Takemori, RIKEN, Yokohama, Japan). PNA was from Vector Laboratories. For intracellular stainings, $\mathrm{B}$ cells where fixed, permeabilized in Cytofix/Cytoperm (BD Biosciences), and stained with IRF4-specific (3E4; BioLegend), H3K27me3-specific
(C36B11; Cell Signaling), or EZH2-specific (AE25.13; K. Helin, University of Copenhagen, Copenhagen, Denmark) $\mathrm{mAb}$ conjugated in house with Alexa Fluor dyes according to manufacturer's instructions (Molecular Probes, Invitrogen). Samples were acquired on a FACSCalibur (BD Biosciences), and data were analyzed with FlowJo software (Tree Star).

Immunoblotting analysis. Immunoblotting analyses were performed with antibodies specific for EZH2 (AE25-13; K. Helin), H3K27me3 (07-449; Upstate), histone H3 (07-690; Millipore), and EED (AA19-30; K. Helin), respectively. Immunoblot images were acquired using the ChemiDoc system (Bio-Rad).

Analysis of apoptosis and DNA damage. GC B cell apoptosis was measured with the CaspGLOW Staining Kit (BioVision). Irradiation was performed using a Faxitron (X-Ray Corporation) on MACS-purified primary B cells stimulated in vitro for 48 hours with $20 \mu \mathrm{g} / \mathrm{ml}$ LPS. TUNEL staining was performed using the In situ Cell Death Detection Kit (Roche) following the manufacturer's instructions. Cells were acquired on a FACSCalibur (BD Biosciences), and data were analyzed with FlowJo software (Tree Star). 53BP $1^{+}$DNA damage foci were quantified in LPS-activated primary B cells 2.5 hours after exposure to 0.2 Gy of IR. To detect 53BP1+ foci, cells were fixed in PBS/4\% paraformaldehyde, permeabilized, and stained with 53BP1-specific antibody (Novus Biologicals, NB100-304), and foci were visualized with a fluorescent-labeled secondary antibody in combination with DAPI staining. Photographs were acquired using an upright fluorescence microscope (Olympus AX-70; $\times 40$ magnification). Cells with 2 or more $53 \mathrm{BP} 1^{+}$foci were counted.

Cell cycle analysis. iGB cells were pulsed with EdU ( $44 \mu \mathrm{M}$; Invitrogen) for 1 hour followed by EdU measurement using the Click-iTEdU Alexa Fluor 647 Flow Cytometry Assay kit according to the manufacturer's instructions (Invitrogen). DLBCL were labeled with BrdU ( $33 \mu \mathrm{M}$; Sigma-Aldrich) for 1 hour followed by determination of BrdU according to manufacturer's instructions (BD Pharmingen). Samples were stained with propidium iodide, acquired on a FACSCalibur (BD Biosciences), and analyzed using FlowJo software (Tree Star).

ELISA. To quantify NP-specific serum antibodies, plates were coated with $2 \mu \mathrm{g} / \mathrm{ml} \mathrm{NP}_{23}$-BSA or $\mathrm{NP}_{4}$-BSA, respectively (Biosearch Technologies), and bound were antibodies detected with biotin-conjugated antiIgM (R33.24.12) or anti-IgG1 (550331; BD Pharmingen) antibodies. IgG1 levels in conditioned media of iGB cultures were measured using a combination of rat anti-mouse IgG1 antibodies (MCA1289 [ABD Serotec] and A85-1 [BD Pharmingen]).

ELISPOT. MultiScreen HTS-HA filter plates (MSHAS4510; Millipore) were coated with $1 \mu \mathrm{g}$ rat anti-mouse IgG1 (LO.MG1-13; ABD Serotec). Cells were seeded for 6 hours at $37^{\circ} \mathrm{C}$, and bound IgG1 antibodies were detected with biotinylated rat anti-mouse IgG1 (A85-1; BD Pharmingen) followed by streptavidin coupled to alkaline phosphatase (ELIspot Kit; $\mathrm{R} \& \mathrm{D}$ Systems). Images of plates were acquired, and the number of spots was scored using ImageJ software.

qPCR analysis. qRT-PCR was performed in triplicate with SYBR Green-I Master Mix using primer combinations listed in Supplemental Table 4 or using custom-made TaqMan Array Micro Fluidic Cards (Applied Biosystems). Probe sets are listed in Supplemental Table 4. Expression values were calculated as $2^{-\Delta c t}$. Ezh2 gene copy number was quantified by qPCR using primers annealing to genomic DNA lying within the loxP-flanked segment (Supplemental Table 4). Values were calculated using the comparative CT method. To normalize for DNA input, a segment of the Gapdh gene was amplified using primers listed in Supplemental Table 4.

Ig $V_{H}$ gene mutation analysis. Ig rearrangements bearing the $V_{H} 186.2$ gene were analyzed as described previously (61). PCR primers are listed in Supplemental Table 4.

Histology. Snap-frozen spleens were embedded in Cryo-M-Bed (Bright Instruments). Sections mounted onto multispot glass slides (Hendley) 
were stained with rat anti-mouse $\operatorname{IgD}$ (BD Biosciences) and biotin-conjugated PNA (Vector Laboratories), followed by rabbit anti-rat Ig coupled to horseradish peroxidase. Sections were washed, and streptavidin complex (Vector Laboratories) was added. Stains were developed with 3,3 diaminobenzidine tetrahydrochloride to reveal horseradish peroxidase-conjugated antibodies and fast blue for biotinylated PNA. Slides were mounted with Immunomount (Thermo) and analyzed with a Leitz Wetzlar Dialux microscope. Photographs were taken with a Leica DM6000 microscope.

ChIP. B cells were fixed in $1 \%(\mathrm{vol} / \mathrm{vol}) \mathrm{PBS} /$ formaldehyde, and the reaction was stopped by adding $0.125 \mathrm{M}$ glycine. Fixed cells were harvested by centrifugation, washed in PBS, and resuspended in lysis buffer $(0.5 \%$ SDS, $50 \mathrm{mM}$ Tris-HCl, $5 \mathrm{mM}$ EDTA, $100 \mathrm{mM} \mathrm{NaCl}$, and $0.02 \% \mathrm{NaN}_{3}$ ) containing $1 \mathrm{mM}$ PMSF and $0.1 \%$ ( $\mathrm{vol} / \mathrm{vol}$ ) protease inhibitor mixture (Sigma-Aldrich). $40 \mu \mathrm{g}$ sonicated chromatin was immunoprecipitated with 3.5 to $5 \mu$ g of antibodies specific for H3 (ab1791; Abcam), H3K27me3 (C36B11; Cell Signaling), and H3K4Me3 (04-745; Millipore) or an IgG control (ab37415; Abcam). Antibodies (3.5-5 $\mu \mathrm{g})$ specific for SUZ12 (D39F6; Cell Signaling) or RNA polymerase-II (ab5131; Abcam) or an IgG control (ab37415; Abcam) were used to immunoprecipitate $500 \mu \mathrm{g}$ chromatin. Antibody-bound DNA was reverse cross-linked, purified, and assayed in triplicate by real-time PCR using primers listed in Supplemental Table 3. ChIP-seq analysis was performed in duplicate on GC B cells sorted from pools of C57BL/ 6 mice immunized with SRBC. $10 \mathrm{ng}$ sonicated chromatin before (input) and after immunoprecipitation with antibodies specific for H3K27me3 (C36B11; Cell Signaling) and H3K4me3 (MC315; Millipore) was processed with the Illumina ChIP-Seq Sample Prep and Multiplexing Oligonucleotide Kits. DNA libraries were quantified and used for cluster generation and sequencing on a HiSeq 2000 instrument (Illumina) following the manufacturer's protocol. ChIP-seq raw data have been deposited in the Gene Expression Omnibus database (GSE50912). Bioinformatic analyses of ChIP-seq data are described in the Supplemental Methods.

Statistics. Statistical analyses were done by 2 -tailed unpaired Student's $t$ test, except for gene expression data using TaqMan Array Cards, for which a nonparametric Wilcoxon rank-sum test was applied. Fisher's exact test was used to assess the enrichment of $\mathrm{H} 3 \mathrm{~K} 27 \mathrm{me} 3$ targets within specific gene lists.
$P$ values equal to or lower than 0.05 were considered indicative of significance. These $P$ values were FDR corrected for multiple comparisons throughout the analysis, with the exception of the identification of enriched chromatin domains (for which much stricter thresholds were applied).

Study approval. Animal experimentation was approved by the IFOM Animal Care Committee (IACUC) and the Italian Ministry of Health.

\section{Acknowledgments}

We thank K. Rajewsky, R. Dalla Favera, and U. Klein for critical reading of the manuscript; $S$. Casola group members and D. Pasini for helpful discussions; and S. Mookherjee and K. Sasmal (InterpretOmics, Bangalore) for support with bioinformatics analysis. We acknowledge the Immgen Consortium for microarray data and the computational research unit of the Center for Genomic Sciences of the Italian Institute of Technology (IIT, Milan) for Illumina sequence data preparation. C. Carrisi was supported by the FIRC. G. Varano was supported by the Umberto Veronesi Foundation. F. Mainoldi was supported by the Association for International Cancer Research. This study was supported by grants from the Italian Association for Cancer Research (to S. Casola), the Italian Ministry of Health (through FIRB IDEAS and the "Progetto Giovani Ricercatori"), and the CARIPLO Foundation (to S. Casola). S. Casola was supported by the FIRC and the Giovanni Armenise-Harvard Foundation.

Received for publication April 23, 2013, and accepted in revised form September 3, 2013.

Address correspondence to: Stefano Casola, IFOM, the FIRC Institute of Molecular Oncology Foundation, Via Adamello 16, 20139, Milan, Italy. Phone: 39.02574303714; Fax: 39.02574303231; E-mail: stefano.casola@ifom.eu.

Marieta Caganova's present address is: Center for Genomic Science of IIT@SEMM, Istituto Italiano di Tecnologia (IIT), Milan, Italy.
1. Victora GD, Nussenzweig MC. Germinal centers. Annu Rev Immunol. 2012;30:429-457.

2. Schwickert TA, et al. A dynamic T cell-limited checkpoint regulates affinity-dependent B cell entry into the germinal center. J Exp Med. 2011; 208(6):1243-1252.

3. Simon JA, Kingston RE. Mechanisms of polycomb gene silencing: knowns and unknowns. Nat Rev Mol Cell Biol. 2009;10(10):697-708.

4. Aldiri I, Vetter ML. PRC2 during vertebrate organogenesis: A complex in transition. Dev Biol. 2012;367(2):91-99.

5. Margueron R, Reinberg D. The Polycomb complex PRC2 and its mark in life. Nature. 2011; 469(7330):343-349.

6. Bernstein BE, et al. A bivalent chromatin structure marks key developmental genes in embryonic stem cells. Cell. 2006;125(2):315-326.

7. Raaphorst FM, et al. Cutting edge: polycomb gene expression patterns reflect distinct B cell differentiation stages in human germinal centers. J Immunol. 2000;164(1):1-4.

8. Velichutina I, et al. EZH2-mediated epigenetic silencing in germinal center B cells contributes to proliferation and lymphomagenesis. Blood. 2010;116(24):5247-5255.

9. Morin RD, et al. Somatic mutations altering EZH2 (Tyr641) in follicular and diffuse large B-cell lymphomas of germinal-center origin. Nat Genet. 2010; 42(2):181-185.

10. Sneeringer CJ, et al. Coordinated activities of wild- type plus mutant EZH2 drive tumor-associated hypertrimethylation of lysine 27 on histone $\mathrm{H} 3$ (H3K27) in human B-cell lymphomas. Proc Natl Acad Sci U S A. 2010;107(49):20980-20985.

11. Su IH, et al. Ezh2 controls B cell development through histone $\mathrm{H} 3$ methylation and Igh rearrangement. Nat Immunol. 2003;4(2):124-131.

12. Casola $S$, et al. Tracking germinal center $B$ cells expressing germ-line immunoglobulin gamma1 transcripts by conditional gene targeting. Proc Natl Acad SciU S A. 2006;103(19):7396-7401.

13. Kraus M, Alimzhanov MB, Rajewsky N, Rajewsky K. Survival of resting mature B lymphocytes depends on BCR signaling via the Igalpha/beta heterodimer. Cell. 2004;117(6):787-800.

14. Bracken AP, et al. The Polycomb group proteins bind throughout the INK4A-ARF locus and are disassociated in senescent cells. Genes Dev. 2007; 21(5):525-530.

15. Smith KG, Light A, O'Reilly LA, Ang SM, Strasser A, Tarlinton D. bcl-2 transgene expression inhibits apoptosis in the germinal center and reveals differences in the selection of memory B cells and bone marrow antibody-forming cells. J Exp Med. 2000; 191(3):475-484.

16. O'Hagan HM, Mohammad HP, Baylin SB. Double strand breaks can initiate gene silencing and SIRT1-dependent onset of DNA methylation in an exogenous promoter CPG island. PLoS Genet. 2008; 4(8):e1000155

17. Liu J, et al. Bmi1 regulates mitochondrial function and the DNA damage response pathway. Nature. 2009;459(7245):387-392.

18. Chou DM, et al. A chromatin localization screen reveals poly (ADP ribose)-regulated recruitment of the repressive polycomb and NuRD complexes to sites of DNA damage. Proc Natl Acad Sci U S A. 2010;107(43):18475-18480.

19. Wu Z, et al. Polycomb protein EZH2 regulates cancer cell fate decision in response to DNA damage. Cell Death Diff. 2011;18(11):1771-1779.

20. Zaheen A, Boulianne B, Parsa JY, Ramachandran S, Gommerman JL, Martin A. AID constrains germinal center size by rendering B cells susceptible to apoptosis. Blood. 2009;114(3):547-554.

21. Fragola $G$, et al. Cell reprogramming requires silencing of a core subset of polycomb targets. PLoS Genet. 2013;9(2):e1003292.

22. Mikkelsen TS, et al. Genome-wide maps of chromatin state in pluripotent and lineage-committed cells. Nature. 2007;448(7153):553-560.

23. Beguelin W, et al. EZH2 is required for germinal center formation and somatic EZH2 mutations promote lymphoid transformation. Cancer Cell. 2013; 23(5):677-692.

24. Chen $\mathrm{H}$, et al. Polycomb protein Ezh2 regulates pancreatic beta-cell Ink4a/Arf expression and regeneration in diabetes mellitus. Genes Dev. 2009; 23(8):975-985.

25. Ezhkova E, Lien WH, Stokes N, Pasolli HA, Silva JM, Fuchs E. EZH1 and EZH2 cogovern histone H3K27 trimethylation and are essential for hair 
follicle homeostasis and wound repair. Genes Dev. 2011;25(5):485-498.

26. Basso K, et al. Integrated biochemical and computational approach identifies BCL6 direct target genes controlling multiple pathways in normal germinal center B cells. Blood. 2010;115(5):975-984.

27. Basso K, Dalla-Favera R. Roles of BCL6 in normal and transformed germinal center B cells. Immunol Rev. 2012;247(1):172-183.

28. Cattoretti G, et al. Deregulated BCL6 expression recapitulates the pathogenesis of human diffuse large B cell lymphomas in mice. Cancer Cell. 2005; $7(5): 445-455$.

29. Nojima T, et al. In-vitro derived germinal centre B cells differentially generate memory B or plasma cells in vivo. Nat Commun. 2011;2:465

30. Pasqualucci L, et al. Inactivation of the PRDM1/ BLIMP1 gene in diffuse large B cell lymphoma. J Exp Med. 2006;203(2):311-317.

31. Calado DP, et al. Constitutive canonical NF-kappaB activation cooperates with disruption of BLIMP1 in the pathogenesis of activated B cell-like diffuse large cell lymphoma. Cancer Cell. 2010;18(6):580-589.

32. Mandelbaum J, et al. BLIMP1 is a tumor suppressor gene frequently disrupted in activated B celllike diffuse large B cell lymphoma. Cancer Cell. 2010;18(6):568-579.

33. McCabe MT, et al. Mutation of A677 in histone methyltransferase EZH2 in human B-cell lymphoma promotes hypertrimethylation of histone $\mathrm{H} 3$ on lysine 27 (H3K27). Proc Natl Acad Sci U S A. 2012; 109(8):2989-2994

34. Verma SK, et al. Identification of potent, selective, cellactive inhibitors of the histone lysine methyltransferase EZH2. ACS Med Chem Lett. 2012;3(12):1091-1096.

35. Knutson SK, et al. A selective inhibitor of EZH2 blocks H3K27 methylation and kills mutant lymphoma cells. Nat Chem Biol. 2012;8(11):890-896.

36. McCabe MT, et al. EZH2 inhibition as a therapeutic strategy for lymphoma with EZH2-activating mutations. Nature. 2012;492(7427):108-112.

37. Qi W, et al. Selective inhibition of Ezh2 by a small molecule inhibitor blocks tumor cells proliferation. Proc Natl Acad Sci U S A. 2012;109(52):21360-21365.

38. O'Hagan HM, et al. Oxidative damage targets com- plexes containing DNA methyltransferases, SIRT1, and polycomb members to promoter CPG Islands. Cancer Cell. 2011;20(5):606-619.

39. Liu M, et al. Two levels of protection for the B cell genome during somatic hypermutation. Nature. 2008;451(7180):841-845

40. Yamane A, et al. Deep-sequencing identification of the genomic targets of the cytidine deaminase AID and its cofactor RPA in B lymphocytes. Nat Immunol. 2011;12(1):62-69.

41. Stanlie A, Aida M, Muramatsu M, Honjo T, Begum NA. Histone 3 lysine 4 trimethylation regulated by the facilitates chromatin transcription complex is critical for DNA cleavage in class switch recombination. Proc Natl Acad SciU S A. 2010;107(51):22190-22195.

42. Kwon K, et al. Instructive role of the transcription factor E2A in early B lymphopoiesis and germinal center B cell development. Immunity. 2008;28(6):751-762.

43. Klein U, et al. Transcription factor IRF4 controls plasma cell differentiation and class-switch recombination. Nat Immunol. 2006;7(7):773-782.

44. Sciammas R, Shaffer AL, Schatz JH, Zhao H, Staudt LM, Singh H. Graded expression of interferon regulatory factor- 4 coordinates isotype switching with plasma cell differentiation. Immunity. 2006;25(2):225-236.

45. Shapiro-Shelef M, Lin KI, McHeyzer-Williams LJ, Liao J, McHeyzer-Williams MG, Calame K. Blimp-1 is required for the formation of immunoglobulin secreting plasma cells and pre-plasma memory B cells. Immunity. 2003;19(4):607-620.

46. Reimold AM, et al. Plasma cell differentiation requires the transcription factor XBP-1. Nature. 2001;412(6844):300-307.

47. Tirosh B, Iwakoshi NN, Glimcher LH, Ploegh HL. XBP-1 specifically promotes IgM synthesis and secretion, but is dispensable for degradation of glycoproteins in primary B cells. J Exp Med. 2005; 202(4):505-516.

48. Ozaki K, et al. A critical role for IL-21 in regulating immunoglobulin production. Science. 2002;298(5598):1630-1634

49. Linterman MA, et al. IL-21 acts directly on B cells to regulate Bcl-6 expression and germinal center responses. J Exp Med. 2010;207(2):353-363.
50. Zotos D, et al. IL-21 regulates germinal center B cell differentiation and proliferation through a B cellintrinsic mechanism. J Exp Med. 2010;207(2):365-378.

51. Kwon H, et al. Analysis of interleukin-21-induced $\operatorname{Prdm} 1$ gene regulation reveals functional cooperation of STAT3 and IRF4 transcription factors. Immunity. 2009;31(6):941-952.

52. Ozaki K, et al. Regulation of B cell differentiation and plasma cell generation by IL-21, a novel inducer of Blimp-1 and Bcl-6. J Immunol. 2004; 173(9):5361-5371.

53. Ochiai K, et al. Transcriptional regulation of germinal center B and plasma cell fates by dynamical control of IRF4. Immunity. 2013;38(5):918-929.

54. Shaffer AL, et al. Blimp-1 orchestrates plasma cell differentiation by extinguishing the mature $\mathrm{B}$ cell gene expression program. Immunity. 2002;17(1):51-62

55. Akamatsu N, et al. High IL-21 receptor expression and apoptosis induction by IL-21 in follicular lymphoma. Cancer Lett. 2007;256(2):196-206.

56. Sarosiek KA, Malumbres R, Nechushtan H, Gentles AJ, Avisar E, Lossos IS. Novel IL-21 signaling pathway up-regulates c-Myc and induces apoptosis of diffuse large B-cell lymphomas. Blood. 2010; 115(3):570-580.

57. Muramatsu M, Kinoshita K, Fagarasan S, Yamada S, Shinkai Y, Honjo T. Class switch recombination and hypermutation require activation-induced cytidine deaminase (AID), a potential RNA editing enzyme. Cell. 2000;102(5):553-563.

58. Serrano M, Lee H, Chin L, Cordon-Cardo C, Beach D, DePinho RA. Role of the INK4a locus in tumor suppression and cell mortality. Cell. 1996;85(1):27-37.

59. Srinivas $\mathrm{S}$, et al. Cre reporter strains produced by targeted insertion of EYFP and ECFP into the ROSA26 locus. BMC Dev Biol. 2001;1:4.

60. Strasser A, et al. Enforced BCL2 expression in B-lymphoid cells prolongs antibody responses and elicits autoimmune disease. Proc Natl Acad Sci US A. 1991;88(19):8661-8665.

61. Esposito G, et al. Mice reconstituted with DNA polymerase beta-deficient fetal liver cells are able to mount a $\mathrm{T}$ cell-dependent immune response and mutate their Ig genes normally. Proc Natl Acad Sci US A. 2000;97(3):1166-1171. 